\title{
Computationally Efficient Nonparametric Importance Sampling
}

\author{
Jan C. Neddermeyer 1
}

Extended Abstract. The variance reduction established by importance sampling strongly depends on the choice of the importance sampling distribution. A good choice is often hard to achieve especially for high-dimensional integration problems. Nonparametric estimation of the optimal importance sampling distribution (known as nonparametric importance sampling) is a reasonable alternative to parametric approaches. In this article nonparametric variants of both the self-normalized and the unnormalized importance sampling estimator are proposed and investigated. A common critique on nonparametric importance sampling is the increased computational burden compared to parametric methods. We solve this problem to a large degree by utilizing the linear blend frequency polygon estimator instead of a kernel estimator. Mean square error convergence properties are investigated leading to recommendations for the efficient application of nonparametric importance sampling. Particularly, we show that nonparametric importance sampling asymptotically attains optimal importance sampling variance. The efficiency of nonparametric importance sampling algorithms heavily relies on the computational efficiency of the employed nonparametric estimator. The linear blend frequency polygon outperforms kernel estimators in terms of certain criteria such as efficient sampling and evaluation. Furthermore, it is compatible with the inversion method for sample generation. This allows to combine our algorithms with other variance reduction techniques such as stratified sampling. Empirical evidence for the usefulness of the suggested algorithms is obtained by means of three benchmark integration problems. As an application we estimate the distribution of the queue length of a spam filter queueing system based on real data.

Keywords. Monte Carlo integration, nonparametric density estimation, multivariate frequency polygon, queueing system, rare event simulation, option pricing.

\footnotetext{
${ }^{1}$ University of Heidelberg, Institute of Applied Mathematics, Im Neuenheimer Feld 294, D-69120 Heidelberg, Germany.
} 


\section{INTRODUCTION}

Importance Sampling (IS) is a general sampling technique for approximating expectations

$$
\mathbf{E}_{p}[\varphi]=I_{\varphi}=\int \varphi(\mathbf{x}) p(\mathbf{x}) d \mathbf{x}
$$

of some function $\varphi: \mathbb{R}^{d} \rightarrow \mathbb{R}$ with respect to a probability density function $p$ on $\mathbb{R}^{d}$. It is often applied if direct sampling from distribution $p$ is computationally too demanding or intractable. But IS is not limited to this purpose. Unless $\varphi$ is constant, IS can often yield massive reduction of the estimators variance if applied carefully. Formally importance sampling is a change of measure. The expectation $\mathbf{E}_{p}[\varphi]$ is rewritten as

$$
\mathbf{E}_{q}[\varphi w]=\int \varphi(\mathbf{x}) w(\mathbf{x}) q(\mathbf{x}) d \mathbf{x}
$$

where $q$ is the probability density function of an importance sampling distribution (also known as proposal) and $w(\mathbf{x})=p(\mathbf{x}) / q(\mathbf{x})$ the Radon-Nikodym derivative. The proposal needs to be chosen so that its support includes the support of $|\phi| p$ or $p$, which imposes a first constraint on $q$. Using importance sampling the integral $I_{\varphi}$ can be estimated by

$$
\hat{I}_{\varphi}^{\mathrm{IS}}=\frac{1}{N} \sum_{i=1}^{N} \varphi\left(\mathbf{x}^{i}\right) w\left(\mathbf{x}^{i}\right),
$$

where $\left\{\mathbf{x}^{1}, \ldots, \mathbf{x}^{N}\right\}$ are drawn from proposal $q$.

In Bayesian inference, it is often the case that either $p$ or the proposal $q$ (or both) are only known up to some constant. In this case an alternative is the self-normalized importance sampling (SIS) estimator given by

$$
\hat{I}_{\varphi}^{\mathrm{SIS}}=\frac{\sum_{i=1}^{N} \varphi\left(\mathbf{x}^{i}\right) w\left(\mathbf{x}^{i}\right)}{\sum_{i=1}^{N} w\left(\mathbf{x}^{i}\right)} .
$$

The strong law of large numbers implies that both $\hat{I}_{\varphi}^{\mathrm{IS}}$ and $\hat{I}_{\varphi}^{\mathrm{SIS}}$ converge almost surely to the expectation $I_{\varphi}$ if it is finite. However, this result is neither of help for assessing the precision of the estimators for a finite set of samples nor for the rate of convergence. In order to construct error bounds it is desirable to have a central limit theorem (CLT) at hand. Under the assumptions that $I_{\varphi}$ and $\operatorname{Var}_{q}[\varphi w]$ are finite a central limit theorem guaranties $\sqrt{N}\left(\hat{I}_{\varphi}^{\mathrm{IS}}-I_{\varphi}\right) \Rightarrow \mathcal{N}\left(0, \sigma_{\mathrm{IS}}^{2}\right)$ where $\sigma_{\mathrm{IS}}^{2}=\mathbf{E}_{q}\left[\varphi w-I_{\varphi}\right]^{2}$ (Rubinstein 1981). The proposal which minimizes the variance $\sigma_{\mathrm{IS}}^{2}$ is given by

$$
q_{\varphi}^{\mathrm{IS}}(\mathbf{x})=\frac{|\varphi(\mathbf{x})| p(\mathbf{x})}{\int|\varphi(\mathbf{x})| p(\mathbf{x}) d \mathbf{x}} .
$$


$q_{\varphi}^{\mathrm{IS}}$ is called the optimal proposal. A CLT for the self-normalised IS estimator $\hat{I}_{\varphi}^{\mathrm{SIS}}$ can be established $\sqrt{N}\left(\hat{I}_{\varphi}^{\text {SIS }}-I_{\varphi}\right) \Rightarrow \mathcal{N}\left(0, \sigma_{\text {SIS }}^{2}\right)$ with limiting variance $\sigma_{\text {SIS }}^{2}=\mathbf{E}_{q}\left[\left(\varphi-I_{\varphi}\right) w\right]^{2}$ under the additional assumption that $\operatorname{Var}_{q}[w]<\infty$ (Geweke 1989). Variance $\sigma_{\text {SIS }}^{2}$ is minimized by the proposal

$$
q_{\varphi}^{\mathrm{SIS}}(\mathbf{x})=\frac{\left|\varphi(\mathbf{x})-I_{\varphi}\right| p(\mathbf{x})}{\int\left|\varphi(\mathbf{x})-I_{\varphi}\right| p(\mathbf{x}) d \mathbf{x}},
$$

provided that the median of $\varphi$ with respect to $p$ exists. The optimal proposals (1) and (2) are merely of conceptual help as the computation of their denominators is typically at least as difficult as the original integration problem. Hence, the objective is to find an easy-to-sample density that approximates the optimal proposals. Traditionally, a proposal is chosen from some parametric family of densities $\left\{q_{\varphi, \theta}, \theta \in \Theta\right\}$ that satisfy the assumptions of the central limit theorems or some related conditions. Typically, it is demanded that the support of $q_{\varphi, \theta}$ includes the support of $|\varphi| p$ or $\left|\varphi-I_{\varphi}\right| p$, respectively, and that the tails of $q$ do not decay faster than those of $|\varphi| p$. Many different density classes have been investigated in the literature including multivariate Student t, mixture, and exponential family distributions (see for instance Geweke 1989; Stadler and Roy 1993; Oh and Berger 1993). The parametrized choice of the proposal can be adaptively revised during the importance sampling which is known as adaptive IS (Oh and Berger 1992; Kollman et al. 1999). Often expectation $I_{\varphi}$ needs to be computed for many different functions $\varphi$ leading to different optimal proposals. As a consequence, it is necessary to investigate the structure of any new $\varphi$ in order to find a suitable parametric family.

A reasonable alternative that does not rely on prior investigation of the structure of the integrand is nonparametric importance sampling (NIS). Nonparametric approximations based on kernel estimators for the construction of proposals have been used before (West 1992, 1993; Givens and Raftery 1996; Kim et al. 2000). Under restrictive conditions it has been shown that nonparametric (unnormalized) IS can not only reduce the variance of the estimator but may also improve its rate of convergence of the mean square error (MSE) to $\mathcal{O}\left(N^{-(d+8) /(d+4)}\right)$ (Zhang 1996). Except for special cases, parametric importance sampling strategies achieve the standard MC rate of $\mathcal{O}\left(N^{-1}\right)$, as the optimal proposal is typically not included in the employed distribution family. There is still a lack of theoretical results for NIS, particularly for the self-normalized importance sampler. Furthermore, computationally aspects, that critically effect the performance of NIS, have only been insufficiently treated in the literature (Zlochin and Baram 2002).

The competitiveness of NIS compared to parametric IS heavily relies on the computational 
efficiency of the employed nonparametric estimator. This article introduces NIS algorithms based on a multivariate frequency polygon estimator which we show to be computationally superior to kernel estimators. Furthermore, our nonparametric estimator allows the combination of NIS with other variance reduction techniques (such as stratified sampling) which is another advantage over kernel estimators. We investigate NIS not only for IS but also for SIS which has not been done before. Under loose conditions on the integrand, the MSE convergence properties of the proposed algorithms are explored. The theoretical findings result in distinct suggestions for efficient application of NIS. The large potential of NIS to reduce MC variance is verified empirically by means of different integration problems. Overall, we provide strong evidence that our NIS algorithms solve well-known problems of existing NIS techniques. This suggests that NIS is a promising alternative to parametric IS in practical applications.

The remainder of the paper is organized as follows. In Sections 2 and 3 we propose NIS algorithms for IS and SIS, respectively, and investigate their MSE convergence properties. In Section 4 we discuss the applicability of the suggested algorithms including parameter selection and implementation issues. Finally, in Section 5 and 6 we present simulation results for three toy integration problems and for a spam filter queueing system based on real data.

\section{NONPARAMETRIC IMPORTANCE SAMPLING}

A NIS algorithm based on a kernel density estimator, that approximates the analytically unavailable optimal proposal $q_{\varphi}^{\mathrm{IS}}$, is considered in Zhang (1996). Theoretical and empirical evidence of the usefulness of this approach has been established. In particular, it was proved that NIS may yield MSE convergence of order $\mathcal{O}\left(N^{-(d+8) /(d+4)}\right)$ essentially under the very restrictive assumption that $\varphi p$ has compact support on which $\varphi$ is strictly positive. The theoretical results derived in this paper get by with much weaker assumptions. From a practical point of view a kernel density estimator is computationally too demanding. For the purpose of NIS it does not suffice that the employed nonparametric estimator provides a fast and accurate approximation of the distribution of interest. It is also required to allow efficient sampling as well as fast evaluation at arbitrary points. As a computationally more efficient alternative to the kernel estimator, it is suggested to use a histogram estimator (Zhang 1996). The drawback of a histogram is its slow convergence rate of $\mathcal{O}\left(N^{-2 /(2+d)}\right)$ compared to kernel estimators, which typically achieve $\mathcal{O}\left(N^{-4 /(4+d)}\right)$. In this paper we propose the usage of a 
multivariate frequency polygon which is known as linear blend frequency polygon (LBFP) (Terrell 1983 cited in Scott 1992, p. 106). It is constructed by interpolation of histogram bin mid-points. Being computationally only slightly more expensive than ordinary histograms, it achieves the same convergence rate as standard kernel estimators. Consider a multivariate histogram estimator with bin height $\hat{f}_{k_{1}, \ldots, k_{d}}^{\mathrm{H}}$ for bin $B_{k_{1}, \ldots, k_{d}}=\prod_{i=1}^{d}\left[t_{k_{i}}-h / 2, t_{k_{i}}+h / 2\right)$ where $h$ is the bin width and $\left(t_{k_{1}}, \ldots, t_{k_{d}}\right)$ the bin mid-point. For $\mathbf{x} \in \prod_{i=1}^{d}\left[t_{k_{i}}, t_{k_{i}}+h\right)$ the LBFP estimator is defined as

$$
\hat{f}(\mathbf{x})=\sum_{j_{1}, \ldots, j_{d} \in\{0,1\}}\left[\prod_{i=1}^{d}\left(\frac{x_{i}-t_{k_{i}}}{h}\right)^{j_{i}}\left(1-\frac{x_{i}-t_{k_{i}}}{h}\right)^{1-j_{i}}\right] \hat{f}_{k_{1}+j_{1}, \ldots, k_{d}+j_{d}}^{\mathrm{H}}
$$

It can be shown that $\hat{f}$ integrates to one.

Our NIS algorithm consists of two steps. In the first step the optimal proposal $q_{\varphi}^{\text {IS }}$ is estimated nonparametrically using samples drawn from a trial distribution $q_{0}$ and weighted according to the importance ratio $q_{\varphi}^{\mathrm{IS}} / q_{0}$. In the second step an ordinary importance sampling is carried out, subject to the proposal estimated in the first step. Before we can state the algorithm we need to introduce the following quantities. Let $A_{M}$ be an increasing sequence of compact sets defined by $A_{M}=\left\{\mathbf{x} \in \mathbb{R}^{d}: q_{\varphi}^{\mathrm{IS}}(\mathbf{x}) \geq c_{M}\right\}$, where $c_{M}>0$ and $c_{M} \rightarrow 0$ as $M$ goes to infinity. For any function $g$ we denote the restriction of $g$ on $A_{M}$ by $g_{M}$ and we abbreviate $q_{M}^{\mathrm{IS}}=q_{\varphi_{M}}^{\mathrm{IS}}$. Furthermore, the volume of $A_{M}$ is denoted by $V_{M}$. Note that, by definition, $A_{M}$ converges to the support of $q_{\varphi}^{\mathrm{IS}}$. The theorems in this section consider the following algorithm, which is related to the NIS algorithm in Zhang (1996).

\section{Algorithm 1 - Nonparametric Importance Sampling}

Step 1: Proposal estimation

- For $j=1, \ldots, M$ : Sample $\tilde{\mathbf{x}}^{j} \sim q_{0}$.

- Obtain estimate $\hat{q}_{M}^{\mathrm{IS}}(\mathbf{x})=\frac{\hat{f}_{M}(\mathbf{x})+\delta_{M}}{\bar{\omega}_{M}+V_{M} \delta_{M}} \mathbf{1}_{A_{M}}(\mathbf{x})$, where $\bar{\omega}_{M}=1 / M \sum_{j=1}^{M} \omega_{M}^{j}, \omega_{M}^{j}=\left|\varphi_{M}\left(\tilde{\mathbf{x}}^{j}\right)\right| p\left(\tilde{\mathbf{x}}^{j}\right) q_{0}\left(\tilde{\mathbf{x}}^{j}\right)^{-1}$, and

$$
\begin{aligned}
\hat{f}_{M}(\mathbf{x})=\frac{1}{M} & \sum_{j_{1}, \ldots, j_{d} \in\{0,1\}}\left[\prod_{i=1}^{d}\left(\frac{x_{i}-t_{k_{i}}}{h}\right)^{j_{i}}\left(1-\frac{x_{i}-t_{k_{i}}}{h}\right)^{1-j_{i}}\right] \\
& \times \sum_{j=1}^{M} \omega_{M}^{j} \mathbf{1}_{\prod_{i=1}^{d}\left[t_{k_{i}}, t_{k_{i}}+h\right)}\left(\tilde{\mathbf{x}}^{j}\right)
\end{aligned}
$$

for $\mathbf{x} \in \prod_{i=1}^{d}\left[t_{k_{i}}, t_{k_{i}}+h\right)$. 
- For $i=1, \ldots, N-M$ : Generate sample $\mathbf{x}^{i}$ from proposal $\hat{q}_{M}^{\mathrm{IS}}$.

- Evaluate $\hat{I}_{\varphi_{M}}^{\mathrm{NIS}}=(N-M)^{-1} \sum_{i=1}^{N-M} \varphi_{M}\left(\mathbf{x}^{i}\right) p\left(\mathbf{x}^{i}\right) \hat{q}_{M}^{\mathrm{IS}}\left(\mathbf{x}^{i}\right)^{-1}$.

Both $A_{M}$ and $\delta_{M}$ are required in the proofs of the Theorems below but they can be omitted in practice.

Assumption 1 Both $\varphi$ and $p$ have three continuous and square integrable derivatives on $\operatorname{supp}(|\varphi| p)$ and $|\varphi| p$ is bounded. Furthermore, $\int\left(\nabla^{2}|\varphi| p\right)^{4}(|\varphi| p)^{-3}<\infty$ where $\nabla^{2}|\varphi| p=$ $\partial^{2}|\varphi| p / \partial x_{1}^{2}+\ldots+\partial^{2}|\varphi| p / \partial x_{d}^{2}$.

Assumption $2 \mathbf{E}\left[|\varphi| p q_{0}^{-1}\right]^{4}$ is finite on $\operatorname{supp}(|\varphi| p)$.

Assumption 3 As total sample size $N \rightarrow \infty$, bin width $h$ satisfies $h \rightarrow 0$ and $M h^{d} \rightarrow \infty$. Additionally, we have $\delta_{M}>0, V_{M} \delta_{M}=o\left(h^{2}\right)$ and $M^{3}\left(V_{M} \delta_{M}\right)^{4} \rightarrow \infty$.

Assumption 4a $c_{M}$ guaranties $\frac{h^{8}+\left(M h^{d}\right)^{-2}}{\delta_{M} c_{M}^{3}}=o\left(\frac{h^{4}+\left(M h^{d}\right)^{-1}}{c_{M}}\right)$ and $\frac{h^{4}+\left(M h^{d}\right)^{-1}}{c_{M}} \rightarrow 0$.

Assumption 5a $c_{M}$ guaranties $\left(\int q_{\varphi}^{\mathrm{IS}} \mathbf{1}_{\left\{q_{\varphi}^{\mathrm{IS}}<c_{M}\right\}}\right)^{2}=o\left(M^{-1} h^{4}+\left(M^{2} h^{d}\right)^{-1}\right)$.

For fixed sample size $M$ and conditional on the samples $\left\{\tilde{\mathbf{x}}^{1}, \tilde{\mathbf{x}}^{2}, \ldots, \tilde{\mathbf{x}}^{M}\right\}$ it is not hard to show that $\hat{I}_{\varphi_{M}}^{\mathrm{NIS}}$ is an unbiased estimator with variance

$$
\operatorname{Var}\left[\hat{I}_{\varphi_{M}}^{\mathrm{NIS}}\right]=\frac{1}{N-M} \int\left(\frac{\varphi_{M}(\mathbf{x}) p(\mathbf{x})}{\hat{q}_{M}^{\mathrm{IS}}(\mathbf{x})}-I_{\varphi_{M}}\right)^{2} \hat{q}_{M}^{\mathrm{IS}}(\mathbf{x}) d \mathbf{x} .
$$

For the special case $\varphi \geq 0$ we have $q_{M}^{\mathrm{IS}}=\varphi_{M} p I_{\varphi_{M}}^{-1}$ and 4 can be rewritten as

$$
\frac{I_{\varphi_{M}}^{2}}{N-M} \int \frac{\left(\hat{q}_{M}^{\mathrm{IS}}(\mathbf{x})-q_{M}^{\mathrm{IS}}(\mathbf{x})\right)^{2}}{\hat{q}_{M}^{\mathrm{IS}}(\mathbf{x})} d \mathbf{x}
$$

Under the foregoing assumptions we now prove that the variance (5) attains convergence rate $\mathcal{O}\left(N^{-(d+8) /(d+4)}\right)$, if bin width $h$ is chosen optimally.

Theorem 1. Suppose Assumptions 1-3, 4a, 5a hold, $\varphi \geq 0$, and $q=q_{\varphi}^{I S}$. We obtain

$$
\mathbf{E}\left[\hat{I}_{\varphi_{M}}^{N I S}-I_{\varphi}\right]^{2}=\frac{I_{\varphi}^{2}}{N-M}\left\{h^{4} H_{1}+\frac{2^{d}}{3^{d} M h^{d}} H_{2}\right\} \times(o(1)+1)
$$

and the optimal bin width

$$
h^{*}=\left(\frac{d H_{2} 2^{d}}{4 H_{1} 3^{d}}\right)^{\frac{1}{d+4}} M^{-\frac{1}{d+4}}
$$

where

$$
H_{1}=\frac{49}{2880} \sum_{i=1}^{d} \int \frac{\left(\partial_{i}^{2} q\right)^{2}}{q}+\frac{1}{64} \sum_{i \neq j} \int \frac{\partial_{i}^{2} q \partial_{j}^{2} q}{q}, H_{2}=\int \frac{q}{q_{0}} .
$$


Proof. See Appendix A.

A direct implication of Theorem 1 is the following corollary.

Corollary 2. Under the assumptions of Theorem 1 and the further assumption that $M / N \rightarrow$ $\lambda(0<\lambda<1)$, and $h=h^{*}$ we yield

$$
\lim _{N \rightarrow \infty} N^{\frac{d+8}{d+4}} \mathbf{E}\left[\hat{I}_{\varphi_{M}}^{N I S}-I_{\varphi}\right]^{2}=\lambda^{-\frac{4}{d+4}}(1-\lambda)^{-1} \times I_{\varphi}^{2} D
$$

and optimal proportion $\lambda^{*}=4 /(d+8)$,

where $D=\left\{(d / 4)^{4 /(d+4)}+(d / 4)^{-d /(d+4)}\right\}\left[H_{1}^{d}\left(2^{d} 3^{-d} H_{2}\right)^{4}\right]^{1 /(d+4)}$.

We remark that under much stronger assumptions corresponding results for NIS based on kernel estimators were obtained in Zhang (1996).

We now move to a more general case. Assume $\varphi \geq 0$ (and $\varphi \leq 0$ ) does not hold. For this case we show that the NIS algorithm asymptotically achieves the minimum importance sampling variance. By substituting the optimal IS distribution $q_{\varphi}^{\mathrm{IS}}$ into variance $\sigma_{\mathrm{IS}}^{2}$ and writing shorthand $\bar{I}_{\varphi}=\int|\varphi(\mathbf{x})| p(\mathbf{x}) d \mathbf{x}$, we see the optimal variance of the IS estimator to be $\bar{I}_{\varphi}^{2}-I_{\varphi}^{2}$.

Assumption $4 \mathbf{b} c_{M}$ guaranties $\frac{h^{8}+\left(M h^{d}\right)^{-2}}{\delta_{M} c_{M}^{5}}=o\left(\frac{h^{4}+\left(M h^{d}\right)^{-1}}{c_{M}^{3}}\right)$ and $\frac{h^{4}+\left(M h^{d}\right)^{-1}}{c_{M}^{3}} \rightarrow 0$.

Assumption $5 \mathbf{b} c_{M}$ guaranties $\left(\int q_{\varphi}^{\mathrm{IS}} \mathbf{1}_{\left\{q_{\varphi}^{\mathrm{IS}}<c_{M}\right\}}\right)^{2}=o\left(M^{-1} h^{2}+\left(M^{2} h^{d}\right)^{-1}\right)$.

Theorem 3. Suppose that Assumptions 1-3, 4b, 5b hold, $\varphi$ does not have a definite sign, and $q=q_{\varphi}^{I S}$. Then we obtain

$$
\mathbf{E}\left[\hat{I}_{\varphi_{M}}^{N I S}-I_{\varphi}\right]^{2}=\frac{1}{N-M}\left[\left(\bar{I}_{\varphi}^{2}-I_{\varphi}^{2}\right)+I_{\varphi}^{2}\left\{h^{2} \bar{H}_{1}+\frac{2^{d}}{3^{d} M h^{d}} \bar{H}_{2}\right\} \times(1+o(1))\right]
$$

and the optimal bin width

$$
h^{* *}=\left(\frac{d \bar{H}_{2} 2^{d-1}}{\bar{H}_{1} 3^{d}}\right)^{\frac{1}{d+2}} M^{-\frac{1}{d+2}}
$$

where $\bar{H}_{1}=-\left(\int f_{\varphi}^{2} \frac{\nabla^{2} q}{8 q^{2}}+\int f_{\varphi} \frac{\nabla^{2} q}{4 q}\right), \bar{H}_{2}=\left(\int \frac{q}{q_{0}}-2 \int \frac{f_{\varphi}}{q_{0}}-\int \frac{f_{\varphi}^{2}}{q_{0} q}\right)$, and $f_{\varphi}=\left(\frac{\varphi p}{I_{\varphi}}-\frac{|\varphi| p}{\bar{I}_{\varphi}}\right)$.

Proof. See Appendix A.

As a consequence of Theorem 3, the NIS algorithm does not lead to a MSE rate improvement for functions $\varphi$ which take positive and negative values. But if the optimal bin width $h^{* *}$ is used, we have

$$
\mathbf{E}\left[\hat{I}_{\varphi_{M}}^{\mathrm{NIS}}-I_{\varphi}\right]^{2}=\frac{\bar{I}_{\varphi}^{2}-I_{\varphi}^{2}}{N-M}+o\left(N^{-1}\right)
$$


That is, the optimal IS variance is achieved asymptotically. Unlike Theorem 1, the optimal proportion $\lambda$ cannot be computed analytically due to its dependency on $N$. But theoretically it can be computed as $\lambda^{* *}=\operatorname{argmin}_{\lambda} G\left(N, h^{* *}, \lambda\right)$ where $G=\mathbf{E}\left[\hat{I}_{\varphi_{M}}^{\mathrm{NIS}}-I_{\varphi}\right]^{2}$. Clearly, $\lambda^{* *}$ decreases in $N$. Note, that for the optimal asymptotic variance to be achieved, it suffices that $0<\lambda<1$.

Corollary 2 and Theorem 3 suggest that IS based Monte Carlo integration can be much more efficient for functions $\varphi \geq 0$ (and $\varphi \leq 0$ ) than for arbitrary functions. This stems from the fact that for non-negative (non-positive) functions the usage of the optimal proposal leads to a zero variance estimator. By approximating the optimal proposal with a consistent estimator it is therefore not surprising that the standard MC rate can be surmounted. Consequently, it should be reasonable to decompose $\varphi$ into positive and negative part, $\varphi=\varphi^{+}-\varphi^{-}$, and apply Algorithm 1 to $\varphi^{+}$and $\varphi^{-}$separately. Since then, we can expect to achieve the superior rate $\mathcal{O}\left(N^{-(d+8) /(d+4)}\right)$. Note that the partitioning of $\varphi$ needs not to be done analytically. It may be carried out implicitly in Step 1 of the algorithm. This approach, denoted by NIS $+/-$, is investigated in a simulation study in Section 5 .

\section{NONPARAMETRIC SELF-NORMALIZED IMPORTANCE SAMPLING}

Many problems in Bayesian inference can be written as the expectation of some function of interest, $\varphi$, with respect to the posterior distribution $p$ which is only known up to some constant. This leads to the evaluation of integrals

$$
\mathbf{E}_{p}[\varphi]=\frac{\int \varphi(\mathbf{x}) \tilde{p}(\mathbf{x}) d \mathbf{x}}{\int \tilde{p}(\mathbf{x}) d \mathbf{x}}
$$

where $\tilde{p}=\alpha p$ with unknown constant $\alpha$. Self-normalized IS is a standard approach for solving such problems. It is often suggested to choose the proposal close to the posterior. But from the CLT we know that one can do better by choosing it close to the optimal proposal which is proportional to $\left|\varphi-I_{\varphi}\right| p$. Next, we introduce a nonparametric self-normalized IS (NSIS) algorithm.

In analogy to the definition of $A_{M}$ we define $\widetilde{A}_{M}=\left\{\mathbf{x} \in \mathbb{R}^{d}: q_{\varphi}^{\mathrm{SIS}}(\mathbf{x}) \geq \tilde{c}_{M}\right\}$, where $\tilde{c}_{M}>0$ and $\tilde{c}_{M} \rightarrow 0$ as $M$ goes to infinity. Its volume is denoted by $\widetilde{V}_{M}$.

\section{Algorithm 2 - Nonparametric Self-Normalized Importance Sampling}

Step 1: Proposal estimation

- For $j=1, \ldots, M$ : Sample $\tilde{\mathbf{x}}^{j} \sim q_{0}$. 
- Obtain estimate $\hat{q}_{M}^{\mathrm{SIS}}(\mathbf{x})=\frac{\hat{f}_{M}(\mathbf{x})+\delta_{M}}{\bar{\omega}_{M}+\widetilde{V}_{M} \delta_{M}} \mathbf{1}_{\widetilde{A}_{M}}(\mathbf{x})$, where $\bar{\omega}_{M}=1 / M \sum_{j=1}^{M} \widetilde{\omega}_{M}^{j}, \widetilde{\omega}_{M}^{j}=\left|\varphi_{M}\left(\tilde{\mathbf{x}}^{j}\right)-\breve{I}_{\varphi_{M}}\right| \tilde{p}\left(\tilde{\mathbf{x}}^{j}\right) q_{0}\left(\tilde{\mathbf{x}}^{j}\right)^{-1}, \hat{f}_{M}(\mathbf{x})$ analogous to Algorithm 1, and

$$
\breve{I}_{\varphi_{M}}=\frac{\sum_{j=1}^{M} \varphi_{M}\left(\tilde{\mathbf{x}}^{j}\right) \tilde{p}\left(\tilde{\mathbf{x}}^{j}\right) q_{0}\left(\tilde{\mathbf{x}}^{j}\right)^{-1}}{\sum_{j=1}^{M} \tilde{p}\left(\tilde{\mathbf{x}}^{j}\right) q_{0}\left(\tilde{\mathbf{x}}^{j}\right)^{-1}} .
$$

Step 2: Self-Normalized Importance Sampling

- For $i=1, \ldots, N-M$ : Generate sample $\mathbf{x}^{i}$ from proposal $\hat{q}_{M}^{\mathrm{SIS}}$.

- Evaluate

$$
\hat{I}_{\varphi_{M}}^{\mathrm{NSIS}}=\frac{\sum_{i=1}^{N-M} \varphi_{M}\left(\mathbf{x}^{i}\right) \widetilde{w}_{M}\left(\mathbf{x}^{i}\right)}{\sum_{i=1}^{N-M} \widetilde{w}_{M}\left(\mathbf{x}^{i}\right)}
$$

where $\widetilde{w}_{M}\left(\mathbf{x}^{i}\right)=\tilde{p}\left(\mathbf{x}^{i}\right) \hat{q}_{M}^{\mathrm{SIS}}\left(\mathbf{x}^{i}\right)^{-1}$.

Both the SIS and NSIS estimator produce biased estimates. But, however, the estimators are asymptotically unbiased. Under Assumptions 1-3 (with $p,|\varphi|, c_{M}, V_{M}$ replaced by $\tilde{p}$, $\left.\left|\varphi-I_{\varphi}\right|, \tilde{c}_{M}, \widetilde{V}_{M}\right)$ it is easy to verify that, conditional on the samples $\left\{\tilde{\mathbf{x}}^{1}, \tilde{\mathbf{x}}^{2}, \ldots, \tilde{\mathbf{x}}^{M}\right\}$, the CLT of Geweke (1989) holds for $\hat{I}_{\varphi_{M}}^{\mathrm{NSIS}}$. The asymptotic variance of the CLT can be written as

$$
\sigma_{\mathrm{SIS}}^{2}=\tilde{I}_{\varphi_{M}}^{2}\left[1+\int \frac{\left(q_{M}^{\mathrm{SIS}}(\mathbf{x})-\hat{q}_{M}^{\mathrm{SIS}}(\mathbf{x})\right)^{2}}{\hat{q}_{M}^{\mathrm{SIS}}(\mathbf{x})} d \mathbf{x}\right]
$$

with $\tilde{I}_{\varphi_{M}}=\int\left|\varphi_{M}(\mathbf{x})-I_{\varphi_{M}}\right| p(\mathbf{x}) d \mathbf{x}$ the median of $\varphi$. Consequently, $\tilde{I}_{\varphi_{M}}^{2}$ is the (asymptotically) optimal variance that can be achieved by self-normalized importance sampling. Unless $\varphi$ is constant, it is impossible to build up a zero variance estimator based on SIS. This renders it unnecessary to investigate separately the MSE convergence of NSIS for non-negative and arbitrary functions.

The structure of $\sigma_{\text {SIS }}^{2}$ is very similar to the structure of the variance in (5) but the weights $\widetilde{\omega}_{M}^{j}$ introduce inter-sample dependencies which make the reasoning in the proofs of Theorem 1 and Theorem 3 not directly applicable. However, similarly to Theorem 3 we can show that the NSIS asymptotically attains optimal variance for certain bin width $h$ and proportion $0<\lambda<1$.

Theorem 4. Suppose that Assumptions 1-3, 4a, 5a (with p, $|\varphi|, c_{M}, V_{M}$ replaced by $\tilde{p}$, $\left.\left|\varphi-I_{\varphi}\right|, \tilde{c}_{M}, \widetilde{V}_{M}\right)$ hold, and $q=q_{\varphi}^{S I S}$. Then we obtain

$$
\mathbf{E}\left[\hat{I}_{\varphi_{M}}^{N S I S}-I_{\varphi}\right]^{2}=\frac{\tilde{I}_{\varphi}^{2}}{N-M}\left[1+h^{4} H_{1}+\frac{2^{d}}{3^{d} M h^{d}} H_{2}\right] \times(1+o(1))
$$


and the optimal bin width

$$
\tilde{h}^{*}=\left(\frac{d H_{2} 2^{d}}{4 H_{1} 3^{d}}\right)^{\frac{1}{d+4}} M^{-\frac{1}{d+4}}
$$

where $\mathrm{H}_{1}$ and $\mathrm{H}_{2}$ are defined as in Theorem 1 (with $q_{\varphi}^{I S}$ replaced by $q_{\varphi}^{\text {SIS }}$ ).

Proof. See Appendix A.

First, note that analogous to Theorem 3 , there is no analytic solution for the optimal $\lambda$. Second, the theorem implies that with NSIS the MSE rate cannot be improved. Therefore, NSIS is (at least asymptotically) less efficient than NIS $+/-$. There is consequently no reason to apply NSIS in cases where NIS $+/-$ is applicable. However, this does not impair the usefulness of NSIS in cases where normalization is required due to unknown constants.

\section{APPLYING NONPARAMETRIC IMPORTANCE SAMPLING}

In this section we discuss what is required for implementing NIS/NSIS. First, one need to take care of the selection of $q_{0}, h$, and $\lambda$. Second, an implementation of the LBFP estimator which allows the generation of samples is required. Given these ingredients the implementation of Algorithm 1 and 2 is straightforward.

\subsection{Parameter Selection}

(i) From a practical point of view trial distribution $q_{0}$ should be chosen such that its support is close to the support of $|\varphi| p$ or $\left|\varphi-I_{\varphi}\right| p$, respectively, and such that it has heavier tails than the corresponding optimal proposal. But it is not required that $q_{0}$ emulates any structure of the optimal proposal. Obviously, the choice should also comply with Assumption 2. Note that the expectations in the assumptions may not exist, if $q_{0}$ is too close to the optimal proposals. In addition, it is important to choose an easy-to-sample density. For low-dimensional problems, even a uniform distribution may suffice.

(ii) As the optimal bin width incorporates unknown quantities dependent on the optimal proposal, it typically cannot be computed analytically. The unknown quantities can be estimated using the plug-in method based on the samples of Step 1 of the algorithms, as suggested in Zhang (1996). If the second derivative of the optimal proposal is unknown, the plug-in method cannot be applied. In this case, a Gaussian reference rule is an alternative. (iii) Except for the case investigated in Theorem 1 and Corollary 2, where the optimal proportion $\lambda^{*}$ is given by a beautifully easy expression only depending on the problem dimension, it is not clear how to choose $\lambda$. However, from the MSE error expressions in the 
theorems we know that $\lambda^{*}$ (from Corollary 2) serves as an upper bound. Empirical evidence suggests that $\lambda$ should never exceed .25.

(iv) In practical applications the restriction of the estimator on a compact set $A_{M}$ can be omitted as the induced bias can be made arbitrarily small and particularly smaller than the desired precision of the integral value. Hence, the sequence $c_{M}$ does not need to be defined. Sequence $\delta_{M}$ can also be skipped in practice as mentioned before.

\subsection{Implementing the LBFP estimator}

The implementation of the LBFP estimator $\hat{f}$ should take into account efficient sampling and evaluation. Given the multivariate histogram defined through bin heights $\hat{f}_{k_{1}, \ldots, k_{d}}^{\mathrm{H}}$ the implementation of the evaluation of $\hat{f}$ is simple (see $(3)$ ). We emphasize that for storing $\hat{f}$ on a computer it suffices to store the underlying histogram. Sampling from a LBFP is more involved than evaluation and to the author's knowledge this has not been discussed in the literature until now. We propose to apply the inversion method. The crucial fact is that a LBFP can be written as a product of (conditional) univariate frequency polygons (FP)

$$
\hat{f}(\mathbf{x})=\hat{f}^{\mathrm{FP}}\left(x_{1}\right) \prod_{i=2}^{d} \hat{f}^{\mathrm{FP}}\left(x_{i} \mid x_{1: i-1}\right)
$$

with $\left\{x_{1: i-1}\right\}=\left\{x_{1}, \ldots, x_{i-1}\right\}$. This representation suggests to produce draws from $\hat{f}$ by sampling recursively from the univariate FPs $\hat{f}^{\mathrm{FP}}$ using their inverse cumulative distribution functions. A FP is a convenient object as it is just a linear interpolated univariate histogram. Furthermore we have

$$
\hat{f}^{\mathrm{FP}}\left(x_{i} \mid x_{1: i-1}\right)=\frac{\hat{f}\left(x_{1: i}\right)}{\hat{f}\left(x_{1: i-1}\right)}
$$

where $\hat{f}\left(x_{1: i}\right)$ are (marginalized) LBFPs, $i=1, \ldots, d$. We will see below that the $\hat{f}^{\mathrm{FP}}\left(x_{i} \mid x_{1: i-1}\right)$ are not required itself but their cumulative distribution functions $\hat{F}\left(x_{i} \mid x_{1: i-1}\right)$. As FPs are piecewise linear functions and due to relation (7) the latter are obtained without difficulty provided that LBFPs $\hat{f}\left(x_{1: i}\right)$ can be evaluated. Hence it is required to calculate the marginalized histograms underlying the LBFPs $\hat{f}\left(x_{1: i}\right)$. These are specified through bins $B_{k_{1}, \ldots, k_{i}}$ and bin heights $\hat{f}_{k_{1}, \ldots, k_{i}}^{\mathrm{H}}$.

Let $\mathbf{y}=\left\{y_{1}, \ldots, y_{d}\right\} \in[0,1)^{d}$ and $y_{i} \in\left[\hat{F}\left(t_{k_{i}} \mid x_{1: i-1}\right), \hat{F}\left(t_{k_{i}+1} \mid x_{1: i-1}\right)\right)$. We now describe how the inverse cumulative distribution functions $\hat{F}^{-1}\left(\cdot \mid x_{1: i-1}\right)$ of $\hat{f}^{\mathrm{FP}}\left(x_{i} \mid x_{1: i-1}\right)$ can be evaluated at $y_{i}$ by making use of $\hat{F}\left(x_{i} \mid x_{1: i-1}\right)$. It is easy to see that, for $x_{i} \in\left[t_{k_{i}}, t_{k_{i}+1}\right), \hat{f}^{\mathrm{FP}}\left(x_{i} \mid x_{1: i-1}\right)$ 
is a linear function with intercept $\alpha$ and slope $\beta$ where

$$
\alpha=\frac{\hat{f}\left(x_{1: i-1}, t_{k_{i}}\right)}{\hat{f}\left(x_{1: i-1}\right)} \quad \text { and } \quad \beta=\frac{1}{h}\left[\frac{\hat{f}\left(x_{1: i-1}, t_{k_{i}+1}\right)}{\hat{f}\left(x_{1: i-1}\right)}-\alpha\right] .
$$

Hence $\hat{F}^{-1}\left(y_{i} \mid x_{1: i-1}\right)$ is the solution of the quadratic equation

$$
y_{i}-\hat{F}\left(t_{k_{i}} \mid x_{1: i-1}\right)=\int_{t_{k_{i}}}^{z} \hat{f}^{\mathrm{FP}}\left(x_{i} \mid x_{1: i-1}\right) d x_{i}=\alpha z+\frac{\beta}{2} z^{2}
$$

which is given by

$$
\hat{F}^{-1}\left(y_{i} \mid x_{1: i-1}\right)= \begin{cases}-\frac{\alpha}{\beta}+\operatorname{sgn}(\beta) \sqrt{\frac{\alpha^{2}}{\beta^{2}}-2 \frac{\gamma_{1}-y_{i}}{\beta}} & \text { for } \beta \neq 0, \\ {\left[\left(\gamma_{2}-y_{i}\right) t_{k_{i}}+\left(y_{i}-\gamma_{1}\right) t_{k_{i}+1}\right] /\left(\gamma_{2}-\gamma_{1}\right)} & \text { for } \beta=0,\end{cases}
$$

where $\gamma_{1}=\hat{F}\left(t_{k_{i}} \mid x_{1: i-1}\right)$ and $\gamma_{2}=\hat{F}\left(t_{k_{i}+1} \mid x_{1: i-1}\right)$.

Summarizing, a sample $\mathbf{x}^{j}$ from the LBFP $\hat{f}$ is obtained through the following recursion. Let $\mathbf{y}^{j}$ be a sample from the uniform distribution on $[0,1)^{d}$. Then, for $i=1, \ldots, d$ :

1. Compute the marginalized histogram associated with LBFP $\hat{f}\left(x_{1: i}\right)$.

2. Calculate cumulative distribution function $\hat{F}\left(x_{i} \mid x_{1: i-1}^{j}\right)$ (or $\hat{F}\left(x_{1}\right)$ for $i=1$ ) at the (marginal) bin mid points $t_{k_{i}}$ using (7).

3. Evaluate $x_{i}^{j}=\hat{F}^{-1}\left(y_{i}^{j} \mid x_{1: i-1}^{j}\right)\left(\right.$ or $x_{1}^{j}=\hat{F}^{-1}\left(y_{1}^{j}\right)$ for $\left.i=1\right)$ using (9).

We remark that for generating $N$ samples Step 1 needs only to be carried out once as it is independent of the particular sample $\mathbf{x}^{j}$. Our $\mathrm{C}++$ implementation of the LBFP is available on request.

\subsection{Computational Remarks}

Now the computational complexity of the LBFP is discussed. For $h=h^{*}$ it can be shown that the complexity for generating $N$ samples from a LBFP is of order $\mathcal{O}\left(2^{d} d^{2} N^{(d+5) /(d+4)}\right)$ (see Appendix B for details). The complexity of evaluation is of lower order. Compared to crude $\mathrm{MC}$ which has $\mathcal{O}(d N)$ sampling from a LBFP is only slightly more expensive for $d$ small. For kernel estimators sampling and evaluation is of order $\mathcal{O}\left(d N^{2}\right)$ (Zlochin and Baram 2002) proving that the LBFP is computationally more efficient for all relevant $d$ and $N$. Note, more efficient sampling from kernel estimates is possible using regularization with whitening (see for instance Musso et al. 2001). But this can induce severe bias if the target distribution is non-Gaussian. 
Besides asymptotic complexity properties there are other computational aspects which are of relevance in practice. On computer systems, the evaluation of functions such as exp and pow is known to be much more expensive than standard arithmetic operations. Contrary to most parametric IS approaches, nonparametric IS methods do not require calls to those functions.

\section{SIMULATIONS}

We consider three toy examples in order to test our nonparametric procedures against (parametric) alternatives. The first two examples are designed to evaluate certain properties of the NIS algorithm and to demonstrate the degraded performance of the NSIS algorithm. The third example is a two-dimensional benchmark problem for self-normalized importance sampling.

A reasonable measure for the effect of a variance reduction technique is the relative efficiency (RE). It is defined as the ratio of the crude MC MSE to the MSE of the method of interest. In the case that both estimators are unbiased, the RE is also known as variance reduction factor. The performance of the different algorithms will be measured by RE and computation time. In all examples the simulation is done for sample sizes $N=1,000, N=$ 5,000 , and $N=10,000$. All computation were carried out on a Dell Precision PWS390, Intel CPU $2.66 \mathrm{GHz}$, and the algorithms are coded in $\mathrm{C}++$. For pseudo random number generation we used the Mersenne Twister 19937 (Matsumoto and Nishimura 1998). All computation times are reported in milliseconds.

Example 1. As our first example we consider a simple integrand that is to be integrated with respect to the standard normal distribution of dimension $d$. The integrand is defined by $\varphi(\mathbf{x})=x_{1} \mathbf{1}_{[-1,1]^{d}}(\mathbf{x})$. It takes positive and negative values on the $d$-dimensional unit cube. This allows the evaluation of the strategy to apply Algorithm 1 separately to the positive and negative part of the integrand ( $\mathrm{NIS}+/-$ ). In our simulation $d$ varies from 1 to 8 . The trial distribution $q_{0}$ is set to the uniform distribution on $[-1,1]^{d}$ and the bin width $h$ is chosen with the plug-in method. $\lambda$ is set to .15 and to the optimal value $4 / 9$ for NIS and $\mathrm{NIS}+/-$, respectively. In order to obtain comparable results, for $\mathrm{NIS}+/-$ the total sample size is equally spread to the integration of the positive and negative part.

Table 1 shows the RE and computation times for MC, NIS, NIS+/-, and ordinary IS (subject to the uniform distribution on $[-1,1]^{d}$ ). The RE figures for $\mathrm{NIS}+/-$ report large 
variance reduction which is present at least up to dimension $d=8$. Even if we take computation time into account, we find significant efficiency improvement: For instance, for $d=4$ and $N=10,000$ we obtain RE of 22 whereas the computation time surplus factor is about 7. Also note, that IS becomes more favorable as $d$ increases. In order to investigate the computationally efficiency we plotted MSE $\times$ computation time (Figure 1). Contrary to RE, smaller values are favourable. We observe that the critical dimension, up to which NIS $+/$ - is computationally more efficient than the other methods, strongly depends on the magnitude of $N$. Whereas for $N=1,000$ one would prefer NIS $+/$ - to IS only for $d=1$, for $N=10,000$ one would do so up to $d=4$. Finally, the convergence of the NIS variance towards the optimal IS variance is examined. The minimum IS variance $\bar{I}_{\varphi}^{2}-I_{\varphi}^{2}$ is approximately .098 and 0.0099 for $d=1,4$, respectively. In Figure 2 the estimated variances of NIS $\times(1-\lambda) N$ are plotted for $100 \leq N \leq 2,500$. The plots indicate rapid convergence to the optimal values. For comparison: the variance of crude $\mathrm{MC} \times N$ is roughly .198 (for $d=1$ ) and .063 (for $d=4$ ).

Example 2. This example is concerned with the pricing of a call option within the BlackScholes model. Given interest rate $r$ and volatility $\sigma$ the evolution of a stock is described by the stochastic differential equation (SDE) $d S(t) / S(t)=r d t+\sigma d W(t)$ with standard Brownian motion $W$. The solution of the SDE is given by $S(T)=S(0) \exp \left[\left(r-0.5 \sigma^{2}\right) T+\right.$ $\sigma \sqrt{T} Z]$ where $Z$ is a standard normal random variable. At time $T$, the call option pays the amount $(S(T)-K)^{+}$depending on the strike level $K$. The price of the option at time 0 is given by the expectation $\mathbf{E}[F(Z)]$ of the discounted payoff $F(Z)=\exp (-r T)(S(T)-K)^{+}$. That is, the pricing problem reduces to the integration of a payoff function with respect to the standard normal distribution. Parametric IS is a standard variance reduction technique for option pricing. A shifted standard normal distribution is often used as proposal. This approach is known as change of drift technique. In our simple model the (asymptotically) optimal drift is given by $\operatorname{argmax}_{z} \log F(z)-.5 z^{2}$ (Glasserman et al. 1999). We state the simulation results for the optimal change of drift IS (CDIS) as parametric benchmark.

For our simulation we set $S(0)=100, r=.1, \sigma=.2, T=1$. The option price is estimated for the strikes $K_{1}=90$ and $K_{2}=130$. For $K_{1}$ the option is said to be in the money $\left(K_{1}<S(0)\right)$ where for $K_{2}$ it is called out-of-the money $\left(K_{2}>S(0)\right)$. The latter case is particularly suited for IS techniques, as crude MC fails to satisfactorily sample into the domain that affects the option price. $q_{0}$ is set to the uniform distribution on $[-5,5]$ and bin width $h$ is selected using the plug-in method. $\lambda$ is set to the optimal value $4 / 9$ for the NIS 
and to .05 for NSIS.

The efficiency improvements of the IS methods relative to crude Monte Carlo integration (RE) are shown in Figure 3. Whereas parametric IS methods and NSIS yield constant reduction factors, NIS realizes increasing relative efficiency which coincides with its theoretical superior convergence rate. Particularly for the out-of-the money scenario, NIS achieves massive variance reduction. Establishing only slight variance reduction NSIS is worst. This confirms our recommendation to avoid NSIS where NIS is applicable. Figure 4 shows the proposals employed in the simulation for strike $K_{2}$. The optimal IS proposal is single-moded and can be reasonably approximated by some Gaussian distribution. This explains the satisfying performance of IS methods based on Gaussian proposals reported in the literature. However, NIS significantly outperforms CDIS. For more complex payoffs implying multimodal optimal proposals, the advantage of NIS should be even more pronounced. Computation times for different sample sizes are reported in Table 2. First, notice that CDIS is much more expensive than MC due to massive evaluation of the exp function whilst computing the likelihood ratios. Second, the computational burden of NIS increases sub-linearly for our sample sizes. This is due to initial computation for the LBFP, which is roughly independent of $N$. Remarkably, NIS is computationally cheaper than CDIS for $N=10,000$.

Example 3. The last example is a two-dimensional benchmark integration problem discussed in Givens and Raftery (1996). The density of interest $p\left(x_{1}, x_{2}\right)$ is given by $X_{1} \sim$ $\mathcal{U}[-1,4]$ and $X_{2} \mid X_{1} \sim \mathcal{N}\left(\left|X_{1}\right|, .09 a^{2}\right)$. We investigate the cases $a=.75$ and $a=3.5$. This kind of density also occurs in work on whale modeling (Raftery 1995). Small values for $a$ imply a strong nonlinear dependency between $X_{1}$ and $X_{2}$. As a becomes larger the dependency vanishes in favor of a more diffuse relationship (see Figure 5). Following Givens and Raftery (1996), we use this scenario for comparing self-normalized IS algorithms. NSIS is tested against SIS with proposal equal to the uniform distribution on $[-4,7] \times[-4,8]$. The same uniform distribution is used as trial distribution $q_{0}$ in the NSIS algorithm. We compute the expectation of functions $\varphi_{1}\left(x_{1}, x_{2}\right)=x_{2}$ and $\varphi_{2}\left(x_{1}, x_{2}\right)=\mathbf{1}_{\left\{x_{1}<0\right\}}\left(x_{1}, x_{2}\right)$. The parameters of NSIS are set as follows: $\lambda=.2$ and $h=1.54,1.224,1.09$ (for $N=1,250,5,000,10,000$ ). For comparison, we also state the results of two other nonparametric algorithms, namely GAIS and LAIS (West 1992; Givens and Raftery 1996). GAIS and LAIS are adaptive nonparametric IS methods, that estimate distribution $p$ with adaptive envelope refinements based on nonparamtric kernel estimators. Density $p$ and the optimal SIS proposals are shown in Figure 5. They are rather far away from the initial guess $q_{0}$. Table 3 shows the relative 
efficiency of NSIS, GAIS, and LAIS with respect to SIS for the two functions and the two different values of $a$. The figures for GAIS and LAIS were reprocessed from Givens and Raftery (1996). For $N=5,000$, NSIS is clearly the method of choice.

\section{APPLICATION}

We investigate a spam filter queueing systems with real data. Queueing system are an active field of research (see for instance Lazowska 1984; Asmussen 2003). Numerous applications are readily available. The most basic queueing system, denoted briefly by $M|M| 1$, consists of a single server and a single waiting room (with infinite capacity). The interarrival and service times of the jobs are exponential distributed with parameter $\mu$ and $\nu$, respectively. This model is well understood theoretically but usually too restrictive for real world applications. In our case, e-mails arrive at a spam filter that decides whether or not a particular e-mail is spam. The data consist of interarrival times $t_{i}$ (in seconds) and service times $s_{i}$ (in milliseconds) for $n=22,248$ e-mails. The data were recorded between 8 am and $8 \mathrm{pm}$ on 8 business days in September 2008 and are available on request. (We are grateful to J. Kunkel for providing the data.) The system that produced the data is a single queue, dual server system, i.e. the e-mails are processed by two parallel spam filter threads. In the following we investigate both the single and the dual server case. The empirical distributions of the interarrival and service times are displayed in Figure 6. We observe that the former is well approximated by an exponential distribution with parameter $\hat{\mu}=n / \sum_{i=1}^{n} t_{i}=.074$ (which is the maximum likelihood estimate). On the contrary, for the service time distribution it is hard to find a parametric model. Therefore we employ a LBFP estimate. (Note that a kernel estimator is inappropriate as heavy sampling from the service time distribution is required.) The bin width was selected with the Gaussian reference rule for frequency polygons $\hat{h}=2.15 \hat{\sigma} n^{-1 / 5}$ (Terrell and Scott 1985) with $\hat{\sigma}$ being the standard deviation of the service times $s_{i}$.

We are interested in the probability that the queue length reaches a certain level $K$. This is a typical problem in queueing systems with rare events being of particular interest. Importance sampling is a standard variance reduction technique for this task (see for instance Glynn and Iglehart 1989; Glasserman and Kou 1995; Kim et al. 2000). For estimating the probabilities we simulate $N$ busy periods and count the number of periods in which level $K$ was reached. A busy period begins when an e-mail has arrived in an empty system and ends when either the system is empty again or the queue length has reached level $K$. Let $\omega_{i}$ be 
the sample path of the queue length in the $i$ th busy period resulting from samples $\mathbf{x}_{i}^{j}$ and $\mathbf{y}_{i}^{k}$ drawn from the interarrival distribution $p_{t}$ and service time distribution $p_{s}$, respectively. In the dual server case $\mathbf{y}_{i}^{k}$ represent the service times of both servers. The MC estimate of the probability of interest is $\hat{I}_{K}=1 / N \sum_{i=1}^{N} \varphi\left(\omega_{i}\right)$ where $\varphi\left(\omega_{i}\right)=1$ if $\omega_{i}$ reaches $K$ and 0 else. Assume the number of e-mails that have been served in the $i$ th busy period is $L_{i}$. Then there must be $K+L_{i}-1$ arrivals in this period for the queue to reach level $K$. (Note, a busy period starts with one job in the queue.) Hence, if importance sampling is used the estimator becomes

$$
\hat{I}_{K}^{\mathrm{IS}}=\frac{1}{N} \sum_{i=1}^{N} \varphi\left(\omega_{i}\right) l\left(\omega_{i}\right)
$$

with likelihood ratio

$$
l\left(\omega_{i}\right)=\prod_{j=1}^{K+L_{i}-1} \frac{p_{t}\left(\mathbf{x}_{i}^{j}\right)}{q_{t}\left(\mathbf{x}_{i}^{j}\right)} \prod_{k=1}^{L_{i}} \frac{p_{s}\left(\mathbf{y}_{i}^{k}\right)}{q_{s}\left(\mathbf{y}_{i}^{k}\right)}
$$

and proposals $q_{t}, q_{s}$. Here NIS works as follows: We simulate $M$ busy periods by sampling interarrival times $\tilde{\mathbf{x}}_{i}^{j}$ and service times $\tilde{\mathbf{y}}_{i}^{k}$ from trial distributions $q_{0, t}$ and $q_{0, s}$, respectively, and obtain sample paths $\tilde{\omega}_{i}, i=1, \ldots, M$. Let $\mathcal{I}=\left\{i \in\{1, \ldots, M\}, \varphi\left(\tilde{\omega}_{i}\right)=1\right\}$. For estimation of the optimal proposals we use those times $\tilde{\mathbf{x}}_{i}^{j}, \tilde{\mathbf{y}}_{i}^{k}$ with $i \in \mathcal{I}$. The interarrival time proposal $\hat{q}_{t}$ is estimated parametrically by using an exponential distribution with parameter

$$
\hat{\mu}=\sum_{i \in \mathcal{I}} \sum_{j=1}^{K+\tilde{L}_{i}-1} w_{i}^{j} / \sum_{i \in \mathcal{I}} \sum_{j=1}^{K+\tilde{L}_{i}-1} w_{i}^{j} \tilde{\mathbf{x}}_{i}^{j}
$$

where $w_{i}^{j}=p_{t}\left(\tilde{\mathbf{x}}_{i}^{j}\right) / q_{0, t}\left(\tilde{\mathbf{x}}_{i}^{j}\right)$. The service time proposal $\hat{q}_{s}$ is estimated nonparametrically (as in Algorithm 1) based on samples $\tilde{\mathbf{y}}_{i}^{k}$ and weights $w_{i}^{k}=p_{s}\left(\tilde{\mathbf{y}}_{i}^{k}\right) / q_{0, s}\left(\tilde{\mathbf{y}}_{i}^{k}\right), i \in \mathcal{I}$.

For our simulation we set $N=1$ Mio.,$\lambda=.15$, and the trial distribution $q_{0, s}$ equal to the LBFP estimate of the service distribution. For $\mathrm{M}|\mathrm{M}| 1$ systems it is well known that (asymptotically) optimal proposals are achieved by swapping the parameters $\mu$ and $\nu$. For this reason $q_{0, t}$ is set to the Exponential distribution with parameter $\hat{\nu}=n / \sum_{i=1}^{n} s_{i}=0.147$. As parametric IS benchmark we consider the IS scheme that carries out IS for the interarrival times only. It uses the Exponential distribution with parameter $\hat{\mu}$ defined in (10) as proposal.

We compare MC, IS, and NIS in terms of the coefficient of variation $(\mathrm{CV})$ and RE. The former is defined as the ratio of the standard deviation to the mean of the probability estimate. Note that for CV smaller values are favourable. The results are summarized in Tables 4 and 5. Where no figure is given, the MC estimator was zero. We find that as the event of interest becomes rarer NIS becomes more favourable. This holds for both the single 
and dual server case. The NIS probability estimates for different queue levels $K$ are shown in Figure 7. No error bounds are given as they are very small for the large number of busy periods used.

Real-world queueing systems typically involve complicated distributions such as the service time distribution in our case. Therefore, it is often impossible to set up parametric IS schemes for simulation. Here, NIS has a distinct advantage. The extension of NIS to the recently proposed state-dependent IS schemes for queueing systems is part of our current research.

\section{CONCLUDING REMARKS}

Contrary to other articles on nonparametric importance sampling, we favored the LBFP instead of kernel estimators. As shown in Section 4, draws from a LBFP can be generated using the inversion method. As the inversion method is a monotone transformation, it preserves the structure of the pre-sampled uniformly distributed variates. This offers the opportunity to combine NIS/NSIS with other variance reduction techniques such as stratified sampling, moment matching, and quasi MC techniques (Robert and Casella 2004; Glasserman 2004).

In financial engineering and many other fields, integration problems are often high-dimensional. Due to the curse of dimensionality and increasing computational complexity, the direct application of NIS is intractable for large dimensions. However, dimension reduction techniques such as principal component analysis, the Brownian Bridge, or the screening method can be applied to break down the required integration task to moderate dimensions (Glasserman 2004; Rubinstein 2007).

Furthermore, we emphasize that the LBFP estimator is not restricted to the usage within nonparametric importance sampling. It is a reasonable alternative to other nonparametric estimators whenever sampling and evaluation is required.

\section{APPENDIX A}

Proof of Theorem 1. We denote $q_{M}^{\mathrm{IS}}$ and $\hat{q}_{M}^{\mathrm{IS}}$ briefly by $q_{M}$ and $\hat{q}_{M}$. Since for $\varphi \geq 0$ we have $q_{M}=\varphi_{M} p I_{\varphi_{M}}^{-1}$, the variance $\sigma_{M}^{2}$ of $\hat{I}_{\varphi_{M}}^{\mathrm{NIS}}$ (conditional on $\left.\left\{\tilde{\mathbf{x}}^{1}, \ldots, \tilde{\mathbf{x}}^{M}\right\}\right)$ is given by

$$
(N-M) \sigma_{M}^{2}=I_{\varphi_{M}}^{2} \int \frac{\left(\hat{q}_{M}(\mathbf{x})-q_{M}(\mathbf{x})\right)^{2}}{\hat{q}_{M}(\mathbf{x})} d \mathbf{x} .
$$

In order to get rid of $\hat{q}_{M}(\mathbf{x})$ in the denominator we write

$$
\begin{aligned}
\frac{N-M}{I_{\varphi_{M}}^{2}} \mathbf{E}\left[\sigma_{M}^{2}\right] & =\mathbf{E}\left[\int \frac{\left(\hat{q}_{M}(\mathbf{x})-q_{M}(\mathbf{x})\right)^{2}}{q_{M}(\mathbf{x})} d \mathbf{x}\right]-\mathbf{E}\left[\int \frac{\left(\hat{q}_{M}(\mathbf{x})-q_{M}(\mathbf{x})\right)^{3}}{\hat{q}_{M}(\mathbf{x}) q_{M}(\mathbf{x})} d \mathbf{x}\right] \\
& =K_{M}+R_{M} .
\end{aligned}
$$


The discrepancy between $\hat{q}_{M}$ and $q_{M}$ can be investigated by

$$
\begin{aligned}
\hat{q}_{M}(\mathbf{x})-q_{M}(\mathbf{x})= & \frac{\hat{f}_{M}(\mathbf{x})-\bar{\omega}_{M} q_{M}(\mathbf{x})}{I_{\varphi_{M}}}+\frac{\delta_{M}\left(1-V_{M} q_{M}(\mathbf{x})\right)}{\bar{\omega}_{M}+V_{M} \delta_{M}} \\
& +\left[\frac{\hat{f}_{M}(\mathbf{x})-\bar{\omega}_{M} q_{M}(\mathbf{x})}{I_{\varphi_{M}}}\right]\left(\frac{I_{\varphi_{M}}}{\bar{\omega}_{M}+V_{M} \delta_{M}}-1\right) \\
= & W_{M}(\mathbf{x})+U_{M}^{1}(\mathbf{x})+U_{M}^{2}(\mathbf{x}) .
\end{aligned}
$$

It will be established below that $\mathbf{E}\left[W_{M}(\mathbf{x})\right]^{2}=O\left(h^{4}+\left(M h^{d}\right)^{-1}\right)$. Now we show that $\mathbf{E}\left[U_{M}^{1}(\mathbf{x})+U_{M}^{2}(\mathbf{x})\right]^{2}$ is of lower order. Under Assumptions 1, 2 we yield

$$
\begin{aligned}
& \mathbf{E}\left[U_{M}^{1}(\mathbf{x})+U_{M}^{2}(\mathbf{x})\right]^{2} \\
& \quad \leq C\left(V_{M} \delta_{M}\right)^{2}+C\left(\mathbf{E}\left[\frac{\hat{f}_{M}(\mathbf{x})-\bar{\omega}_{M} q_{M}(\mathbf{x})}{I_{\varphi_{M}}}\right]^{4}\right)^{1 / 2}\left(\mathbf{E}\left[\frac{I_{\varphi_{M}}}{\bar{\omega}_{M}+V_{M} \delta_{M}}-1\right]^{4}\right)^{1 / 2} \\
& \leq C\left(V_{M} \delta_{M}\right)^{2}+C\left(\frac{1}{M h^{d}}+h^{4}\right)\left(\frac{1}{M^{3}\left(V_{M} \delta_{M}\right)^{4}}+\left(V_{M} \delta_{M}\right)^{2}+\frac{1}{M^{2}}\right)^{1 / 2} .
\end{aligned}
$$

The last inequality follows analogously to Lemma 1, 2 in Zhang (1996). Since by Assumption $3 V_{M} \delta_{M}=o\left(h^{2}\right)$ and $M^{3}\left(V_{M} \delta_{M}\right)^{4} \rightarrow \infty$, we obtain $\mathbf{E}\left[U_{M}^{1}(\mathbf{x})+U_{M}^{2}(\mathbf{x})\right]^{2}=o\left(\mathbf{E}\left[W_{M}(\mathbf{x})\right]^{2}\right)$. We conclude $K_{M} \approx \int \mathbf{E}\left[W_{M}(\mathbf{x})^{2}\right] q_{M}^{-1}(\mathbf{x}) d \mathbf{x}$.

It is not hard to work out that $\int \mathbf{E}\left[W_{M}(\mathbf{x})^{2}\right] q_{M}^{-1}(\mathbf{x}) d \mathbf{x}$ decomposes into an integrated squared bias term $L_{1}$ and an integrated variance term $L_{2}$

$$
\int \frac{\left(\mathbf{E}\left[\hat{f}_{M}(\mathbf{x}) I_{\varphi_{M}}^{-1}\right]-q_{M}(\mathbf{x})\right)^{2}}{q_{M}(\mathbf{x})} d \mathbf{x}+\int \frac{\operatorname{Var}\left[\hat{f}_{M}(\mathbf{x}) I_{\varphi_{M}}^{-1}\right]}{q_{M}(\mathbf{x})} d \mathbf{x}+O\left(M^{-1}\right)=L_{1}+L_{2}+O\left(M^{-1}\right) .
$$

For notational convenience the following is only shown for $d=1$. Without loss of generality we assume $\mathbf{x} \in[-h / 2, h / 2)$. Then $\hat{f}_{M} I_{\varphi_{M}}^{-1}$ simplifies to

$$
\frac{\hat{f}_{M}(\mathbf{x})}{I_{\varphi_{M}}}=\left(\frac{h / 2-\mathbf{x}}{h}\right) \frac{\hat{f}_{0}^{\mathrm{UH}}}{I_{\varphi_{M}}}+\left(\frac{h / 2+\mathbf{x}}{h}\right) \frac{\hat{f}_{1}^{\mathrm{UH}}}{I_{\varphi_{M}}}
$$

where $\hat{f}_{0}^{\mathrm{UH}}=1 / M \sum_{j=1}^{M} \omega_{M}^{j} \mathbf{1}_{[-h, 0)}\left(\tilde{\mathbf{x}}^{j}\right)$ and $\hat{f}_{1}^{\mathrm{UH}}=1 / M \sum_{j=1}^{M} \omega_{M}^{j} \mathbf{1}_{[0, h)}\left(\tilde{\mathbf{x}}^{j}\right)$ are the heights of bins $[-h, 0)$, respectively, $[0, h)$ of an unnormalized histogram. For the computation of $L_{1}$ we need to compare the Taylor expansions of $\mathbf{E}\left[\hat{f}_{M}(\mathbf{x}) I_{\varphi_{M}}^{-1}\right]$ and $q_{M}$ which are given by

$$
\begin{aligned}
\mathbf{E}\left[\hat{f}_{M}(\mathbf{x}) I_{\varphi_{M}}^{-1}\right] & =q_{M}(0)+\mathbf{x} q_{M}^{\prime}(0)+h^{2} q_{M}^{\prime \prime}(0) / 6+O\left(h^{3}\right) \\
q_{M}(\mathbf{x}) & =q_{M}(0)+\mathbf{x} q_{M}^{\prime}(0)+\mathbf{x}^{2} q_{M}^{\prime \prime}(0) / 2+O\left(h^{3}\right)
\end{aligned}
$$

The former follows from (13) and from the expansion of the histogram

$$
\mathbf{E}\left[\hat{f}_{0 / 1}^{\mathrm{UH}} I_{\varphi_{M}}^{-1}\right]=q_{M}(0)-/+h q_{M}^{\prime}(0) / 2+h^{2} q_{M}^{\prime \prime}(0) / 6+O\left(h^{3}\right)
$$


Thus we obtain $\left(\mathbf{E}\left[\hat{f}_{M}(\mathbf{x}) I_{\varphi_{M}}^{-1}\right]-q_{M}(\mathbf{x})\right)^{2} \approx\left(h^{2}-3 \mathbf{x}^{2}\right)^{2} q_{M}^{\prime \prime}(0)^{2} / 36$. Integration over $[-h / 2, h / 2)$ and using Taylor expansion of $1 / q_{M}(\mathbf{x})$ about 0 leads to

$$
\frac{q_{M}^{\prime \prime}(0)^{2}}{36} \int_{-h / 2}^{h / 2} \frac{\left(h^{2}-3 \mathbf{x}^{2}\right)^{2}}{q_{M}(\mathbf{x})} d \mathbf{x}=\frac{49 q_{M}^{\prime \prime}(0)^{2}}{2880 q_{M}(0)} h^{5}+O\left(h^{6}\right) .
$$

By summing over all bins and applying standard Riemann approximation we yield

$$
L_{1}=\frac{49}{2880} h^{4} \int \frac{q_{M}^{\prime \prime}(\mathbf{x})^{2}}{q_{M}(\mathbf{x})} d \mathbf{x}+O\left(h^{5}\right) .
$$

Next we derive an approximation to $L_{2}$. From $(13)$ we have

$$
\begin{aligned}
\operatorname{Var}\left[\hat{f}_{M}(\mathbf{x}) I_{\varphi_{M}}^{-1}\right]= & \left(\frac{h / 2-\mathbf{x}}{h}\right)^{2} \operatorname{Var}\left[\hat{f}_{0}^{\mathrm{UH}} I_{\varphi_{M}}^{-1}\right]+\left(\frac{h / 2+\mathbf{x}}{h}\right)^{2} \operatorname{Var}\left[\hat{f}_{1}^{\mathrm{UH}} I_{\varphi_{M}}^{-1}\right] \\
& +\frac{h^{2} / 2-2 \mathbf{x}^{2}}{h^{2}} \operatorname{Cov}\left[\hat{f}_{0}^{\mathrm{UH}} I_{\varphi_{M}}^{-1}, \hat{f}_{1}^{\mathrm{UH}} I_{\varphi_{M}}^{-1}\right] .
\end{aligned}
$$

In addition, it can be shown that $\operatorname{Var}\left[\hat{f}_{i}^{\mathrm{UH}} I_{\varphi_{M}}^{-1}\right] \approx \frac{q_{M}(0)^{2}}{M h q_{0}(0)}-\frac{q_{M}(0)^{2}}{M}$ for $i=0,1$ and $\operatorname{Cov}\left[\hat{f}_{0}^{\mathrm{UH}} I_{\varphi_{M}}^{-1}, \hat{f}_{1}^{\mathrm{UH}} I_{\varphi_{M}}^{-1}\right] \approx$ $-\frac{q_{M}(0)^{2}}{M}$ analogously to Scott $(1992$, chap. 4). That is we yield

$$
\operatorname{Var}\left[\hat{f}_{M}(\mathbf{x}) I_{\varphi_{M}}^{-1}\right]=\left(\frac{1}{2 M h}+\frac{2 \mathbf{x}^{2}}{M h^{3}}\right) \frac{q_{M}(0)^{2}}{q_{0}(0)}+O\left(M^{-1}\right) .
$$

Analogously to (14) and 15 we then obtain

$$
\int_{-h / 2}^{h / 2} \frac{\operatorname{Var}\left[\hat{f}_{M}(\mathbf{x}) I_{\varphi_{M}}^{-1}\right]}{q_{M}(\mathbf{x})} d \mathbf{x}=\frac{2 q_{M}(0)}{3 M q_{0}(0)}+O(h / M)
$$

and

$$
L_{2}=\frac{2}{3 M h} \int \frac{q_{M}(\mathbf{x})}{q_{0}(\mathbf{x})} d \mathbf{x}+O\left(M^{-1}\right),
$$

respectively. Very similar computations in the multivariate case yield

$$
K_{M} \approx h^{4} H_{M, 1}+\frac{2^{d}}{3^{d} M h^{d}} H_{M, 2}
$$

where

$$
H_{M, 1}=\frac{49}{2880} \sum_{i=1}^{d} \int \frac{\left(\partial_{i}^{2} q_{M}\right)^{2}}{q_{M}}+\frac{1}{64} \sum_{i \neq j} \int \frac{\partial_{i}^{2} q_{M} \partial_{j}^{2} q_{M}}{q_{M}} \quad \text { and } \quad H_{M, 2}=\int \frac{q_{M}}{q_{0}} .
$$

It remains to show that $R_{M}$ is negligible compared to $K_{M}$. The construction of $\hat{g}_{M}$ implies $\hat{g}_{M} \geq \delta_{M}\left(\bar{\omega}_{M}+V_{M} \delta_{M}\right)^{-1}>0$. Under Assumption 1 and 4 a it can be shown that $R_{M}$ is negligible following the same lines as in Zhang (1996). 
The proof is finished by noting that the squared bias term in $\mathbf{E}\left[\hat{I}_{\varphi_{M}}^{\mathrm{NIS}}-I_{\varphi}\right]^{2}$ is negligible due to Assumption 5a and that the expressions $I_{\varphi_{M}}^{2}$ (in $(11)$ ), $H_{M, 1}$, and $H_{M, 2}$ can be substituted by their unrestricted counterparts as their differences are of lower order.

Proof of Theorem 3. Again $q_{M}$ is shorthand for $q_{M}^{\mathrm{IS}}$. Let $f_{\varphi_{M}}=\left(\frac{\varphi_{M} p}{I_{\varphi_{M}}}-\frac{\left|\varphi_{M}\right| p}{\bar{I}_{\varphi_{M}}}\right)$. Straight forward calculations yield

$$
\begin{aligned}
(N-M) \sigma_{M}^{2} & =I_{\varphi_{M}}^{2} \int\left(\frac{\varphi_{M} p}{I_{\varphi_{M}}}-\frac{\left|\varphi_{M}\right| p}{\bar{I}_{\varphi_{M}}}+q_{M}-\hat{q}_{M}\right)^{2} \hat{q}_{M}^{-1} \\
& =I_{\varphi_{M}}^{2}\left[\int f_{\varphi_{M}}^{2} \frac{\left(q_{M}-\hat{q}_{M}\right)}{q_{M} \hat{q}_{M}}+2 \int f_{\varphi_{M}} \frac{\left(q_{M}-\hat{q}_{M}\right)}{\hat{q}_{M}}+\int \frac{\left(q_{M}-\hat{q}_{M}\right)^{2}}{\hat{q}_{M}}+\int \frac{f_{\varphi_{M}}^{2}}{q_{M}}\right] \\
& =I_{\varphi_{M}}^{2}\left[T_{1}+T_{2}+T_{3}+T_{4}\right] .
\end{aligned}
$$

Term $T_{4}$ is independent of the nonparametric estimation and we have $I_{\varphi_{M}}^{2} T_{4}=\bar{I}_{\varphi_{M}}^{2}-I_{\varphi_{M}}^{2}$. The expectation of term $T_{1}$ can be written as

$$
\int f_{\varphi_{M}}^{2} \frac{\mathbf{E}\left[q_{M}-\hat{q}_{M}\right]}{q_{M}^{2}}-\int f_{\varphi_{M}}^{2} \frac{\mathbf{E}\left[q_{M}-\hat{q}_{M}\right]^{2}}{q_{M}^{3}}+\int f_{\varphi_{M}}^{2} \frac{\mathbf{E}\left[q_{M}-\hat{q}_{M}\right]^{3}}{q_{M}^{3} \hat{q}_{M}}=T_{1,1}+T_{1,2}+T_{1,3} .
$$

Similar expressions are obtained for quantities $T_{2}$ and $T_{3}$. We begin with $T_{1,1}$. Analogously to 12 we conclude $q_{M}(\mathbf{x})-\hat{q}_{M}(\mathbf{x}) \approx-\left[\hat{f}_{M}(\mathbf{x})-\bar{\omega}_{M} q_{M}(\mathbf{x})\right] / \bar{I}_{\varphi_{M}}$. From the proof of Theorem 1 we also know that

$$
\mathbf{E}\left[\hat{f}_{M}(\mathbf{x})-\bar{\omega}_{M} q_{M}(\mathbf{x}) / \bar{I}_{\varphi_{M}}\right]=\mathbf{E}\left[\hat{f}_{M}(\mathbf{x}) \bar{I}_{\varphi_{M}}^{-1}\right]-q_{M}(\mathbf{x})=\left(h^{2}-3 \mathbf{x}^{2}\right) q_{M}^{\prime \prime}(0) / 6+O\left(h^{3}\right)
$$

for $d=1$ and $\mathbf{x} \in[-h / 2, h / 2)$. Then we obtain

$$
\frac{q_{M}^{\prime \prime}(0)}{6} \int_{-h / 2}^{h / 2} f_{\varphi_{M}}(\mathbf{x})^{2} \frac{h^{2}-3 \mathbf{x}^{2}}{q_{M}(\mathbf{x})^{2}} d \mathbf{x}=\frac{h^{3}}{8} f_{\varphi_{M}}(0)^{2} \frac{q_{M}^{\prime \prime}(0)}{q_{M}(0)^{2}}+O\left(h^{4}\right)
$$

using a Taylor expansion of $f_{\varphi_{M}}(\mathbf{x})^{2} / q_{M}(\mathbf{x})^{2}$ about 0 . Finally, summing over all bins and using Riemann approximation gives $T_{1,1}$ in the one-dimensional case

$$
-\frac{h^{2}}{8} \int f_{\varphi_{M}}(\mathbf{x})^{2} \frac{q_{M}^{\prime \prime}(\mathbf{x})}{q_{M}(\mathbf{x})^{2}} d \mathbf{x}+O\left(h^{3}\right)
$$

In the multivariate case we yield

$$
T_{1,1}=-\frac{h^{2}}{8} \int f_{\varphi_{M}}^{2}(\mathbf{x}) \frac{\nabla^{2} q_{M}(\mathbf{x})}{q_{M}(\mathbf{x})^{2}} d \mathbf{x}+O\left(h^{3}\right) .
$$

Term $T_{1,2}$ can be treated analogously to $\int \mathbf{E}\left[W_{M}(\mathbf{x})^{2}\right] q_{M}^{-1}(\mathbf{x}) d \mathbf{x}$ in the proof of Theorem 1 . We end up with

$$
T_{1,2}=-\frac{2^{d}}{3^{d} M h^{d}} \int \frac{f_{\varphi_{M}}^{2}}{q_{0} q_{M}}-\left[\frac{49 h^{4}}{2880} \sum_{i=1}^{d} \int f_{\varphi_{M}}^{2} \frac{\left(\partial_{i}^{2} q_{M}\right)^{2}}{q_{M}^{3}}+\frac{h^{4}}{64} \sum_{i \neq j} \int f_{\varphi_{M}}^{2} \frac{\partial_{i}^{2} q_{M} \partial_{j}^{2} q_{M}}{q_{M}^{3}}\right] .
$$


Comparing the term in brackets to $T_{1,1}$ we observe that the former is negligible. Furthermore, similarly to $R_{M}$ in the proof of Theorem 1 , it follows that $T_{1,3}$ is negligible compared to $T_{1,2}$ provided that Assumption 4 holds.

The calculations for $T_{2}$ and $T_{3}$ are very similar to those of $T_{1}$ and therefore omitted. Putting all terms together we obtain

$$
\begin{aligned}
(N-M) \mathbf{E}\left[\sigma_{M}^{2}\right]= & I_{\varphi_{M}}^{2}\left\{\frac{2^{d}}{3^{d} M h^{d}}\left(\int \frac{q_{M}}{q_{0}}-2 \int \frac{f_{\varphi_{M}}}{q_{0}}-\int \frac{f_{\varphi_{M}}^{2}}{q_{0} q_{M}}\right)\right. \\
& \left.-h^{2}\left(\int f_{\varphi_{M}}^{2} \frac{\nabla^{2} q_{M}}{8 q_{M}^{2}}+\int f_{\varphi} \frac{\nabla^{2} q_{M}}{4 q_{M}}\right)\right\} \times(1+o(1))+\left(\bar{I}_{\varphi_{M}}^{2}-I_{\varphi_{M}}^{2}\right) .
\end{aligned}
$$

We observe that the terms restricted on $M$ can be substituted by their asymptotic limits, which completes the proof.

Proof of Theorem 4. We denote $q_{M}^{\mathrm{SIS}}$ and $\hat{q}_{M}^{\mathrm{SIS}}$ briefly by $q_{M}$ and $\hat{q}_{M}$. As the bias of $\hat{I}_{\varphi_{M}}^{\mathrm{NSIS}}$ is asymptotically negligible we have $\mathbf{E}\left[\hat{I}_{\varphi_{M}}^{\mathrm{NSIS}}-I_{\varphi}\right]^{2}=(N-M)^{-1} \mathbf{E}\left[\sigma_{\mathrm{SIS}}^{2}\right] \times\{1+o(1)\}$. Thus, it suffices to examine $\mathbf{E}\left[\sigma_{\text {SIS }}^{2}\right]$ with $\sigma_{\text {SIS }}^{2}$ as in (6). We obtain analogously to 12

$$
\hat{q}_{M}(\mathbf{x})-q_{M}(\mathbf{x})=\frac{\hat{f}_{M}(\mathbf{x})-\bar{\omega}_{M} q_{M}(\mathbf{x})}{\alpha \tilde{I}_{\varphi_{M}}}+\widetilde{U}_{M}^{1}(\mathbf{x})+\widetilde{U}_{M}^{2}(\mathbf{x})
$$

The crucial step for proving that the remainder term $\widetilde{U}_{M}^{1}(\mathbf{x})+\widetilde{U}_{M}^{2}(\mathbf{x})$ is of lower order is to show that under Assumptions 1, 2

$$
\mathbf{E}\left[\alpha \tilde{I}_{\varphi_{M}}-\bar{\omega}_{M}\right]^{2 l} \leq C M^{-l}
$$

for $l=1,2$ (compare Lemma 1, 2 in Zhang (1996)). We have

$$
\begin{aligned}
\left|\alpha \tilde{I}_{\varphi_{M}}-\bar{\omega}_{M}\right| \leq\left|\alpha \tilde{I}_{\varphi_{M}}-\frac{1}{M} \sum_{j=1}^{M}\right| \varphi_{M}\left(\tilde{\mathbf{x}}^{j}\right)-I_{\varphi_{M}}\left|\tilde{p}\left(\tilde{\mathbf{x}}^{j}\right) q_{0}\left(\tilde{\mathbf{x}}^{j}\right)^{-1}\right| \\
+\frac{1}{M} \sum_{j=1}^{M} \tilde{p}\left(\tilde{\mathbf{x}}^{j}\right) q_{0}\left(\tilde{\mathbf{x}}^{j}\right)^{-1}\left|I_{\varphi_{M}}-\breve{I}_{\varphi_{M}}\right|
\end{aligned}
$$

and by applying the Minkowski inequality we obtain

$$
\begin{aligned}
\left(\mathbf{E}\left[\alpha \tilde{I}_{\varphi_{M}}-\bar{\omega}_{M}\right]^{2 l}\right)^{\frac{1}{2 l} \leq} & \left(\mathbf{E}\left[\alpha \tilde{I}_{\varphi_{M}}-\frac{1}{M} \sum_{j=1}^{M}\left|\varphi_{M}\left(\tilde{\mathbf{x}}^{j}\right)-I_{\varphi_{M}}\right| \tilde{p}\left(\tilde{\mathbf{x}}^{j}\right) q_{0}\left(\tilde{\mathbf{x}}^{j}\right)^{-1}\right]^{2 l}\right)^{\frac{1}{2 l}} \\
& +C\left(\mathbf{E}\left[I_{\varphi_{M}}-\breve{I}_{\varphi_{M}}\right]^{2 l}\right)^{\frac{1}{2 l}} \\
= & C\left(M^{-1 / 2}+M^{-1 / 2}\right) .
\end{aligned}
$$


Hence, we conclude that the remainder term is of lower order. Finally, we need to show that

$$
\int \mathbf{E}\left[\left(\frac{\hat{f}_{M}(\mathbf{x})-\bar{\omega}_{M} q_{M}(\mathbf{x})}{\alpha \tilde{I}_{\varphi_{M}}}\right)^{2} \hat{q}_{M}(\mathbf{x})^{-1}\right] d \mathbf{x} \approx h^{4} H_{1}+\frac{2^{d}}{3^{d} M h^{d}} H_{2}
$$

The main difference to Theorem 1 is the dependency of the weights $\widetilde{\omega}_{M}^{j}$. Define $\check{\omega}_{M}^{j}=$ $\left|\varphi_{M}\left(\tilde{\mathbf{x}}^{j}\right)-I_{\varphi_{M}}\right| \tilde{p}\left(\tilde{\mathbf{x}}^{j}\right) q_{0}\left(\tilde{\mathbf{x}}^{j}\right)^{-1}, j=1, \ldots, M$. As in the proof of Theorem 1 let $\tilde{f}_{0 / 1}^{\mathrm{UH}}$ and $\hat{f}_{0 / 1}^{\mathrm{UH}}$ be unnormalized histogram bins based on weights $\check{\omega}_{M}^{j}$ and $\widetilde{\omega}_{M}^{j}$, respectively. It is not hard to show that $\mathbf{E}\left[\hat{f}_{0 / 1}^{\mathrm{UH}}\left(\alpha \tilde{I}_{\varphi_{M}}\right)^{-1}\right]=\mathbf{E}\left[\tilde{f}_{0 / 1}^{\mathrm{UH}}\left(\alpha \tilde{I}_{\varphi_{M}}\right)^{-1}\right]+\mathcal{O}\left(M^{-1 / 2}\right)$ and $\operatorname{Var}\left[\hat{f}_{0 / 1}^{\mathrm{UH}}\left(\alpha \tilde{I}_{\varphi_{M}}\right)^{-1}\right]=$ $\operatorname{Var}\left[\check{f}_{0 / 1}^{\mathrm{UH}}\left(\alpha \tilde{I}_{\varphi_{M}}\right)^{-1}\right]+\mathcal{O}\left(M^{-1}\right)$. The rest of the proof follows analogously to Theorem 1 since weights $\check{\omega}_{M}^{j}$ are independent and the additional $\mathcal{O}\left(M^{-1 / 2}\right), \mathcal{O}\left(M^{-1}\right)$ terms are negligible.

\section{APPENDIX B}

Let $B_{M}$ be the number of bins. It follows that the number of bins in each marginal space is $\mathcal{O}\left(B_{M}^{1 / d}\right)$. We begin with the analysis of the evaluation of a LBFP. Given location $\mathbf{x}$ we need to find the associated bin mid-points $\left(t_{k_{1}}, \ldots, t_{k_{d}}\right)$ which is of order $\mathcal{O}\left(d B_{M}^{1 / d}\right)$. Then formula (3) can be evaluated which is $\mathcal{O}\left(2^{d} d\right)$. Now observe $B_{M} \approx V_{M} / h^{d}$ and $h^{*}=$ $\mathcal{O}\left(\rho(d)^{1 /(d+4)} N^{-1 /(d+4)}\right)$ with $\rho(d)=d(2 / 3)^{d}$. By assuming that $h=h^{*}$ we obtain $\mathcal{O}\left(d B_{M}^{1 / d}+\right.$ $\left.2^{d} d\right) \approx \mathcal{O}\left(\rho(d)^{-1 /(d+4)} d N^{1 /(d+4)}+2^{d} d\right)$ neglecting the slowly increasing sequence $V_{M}$.

Sampling from a LBFP consists of the three steps described in Section 4.2. In Step 1 the marginalized histograms corresponding to the $\operatorname{LBFP} \hat{f}\left(x_{1: i}\right), i=1, \ldots, d-1$, need to be calculated. This can be done recursively in $\mathcal{O}\left(B_{M}\right)$. In the second step $\hat{F}$ are to be computed at all bin mid-points $t_{k_{i}}$ using relation (7). Thus it is required to evaluate $\hat{f}\left(x_{1: i-1}, t_{k_{i}}\right), i=1, \ldots, d$. This consists of searching the bin mid-points $\left(t_{k_{1}}, \ldots, t_{k_{i-1}}\right)$ associated with $x_{1: i-1}$ and evaluating formula (3) as we have discussed above. It is sufficient to do the former once. Thus we end up with $\mathcal{O}\left(d B_{M}^{1 / d}+2^{d} d \times d B_{M}^{1 / d}\right)$ where the latter $d B_{M}^{1 / d}$ is due to the evaluation of $\hat{F}$ at all $t_{k_{i}}$ in each marginal dimension. Step 3 has complexity $\mathcal{O}\left(d B_{M}^{1 / d}\right)$ as in each marginal dimension the bin mid-point $t_{k_{i}}$ satisfying $y_{i} \in$ $\left[\hat{F}\left(t_{k_{i}} \mid x_{1: i-1}\right), \hat{F}\left(t_{k_{i}+1} \mid x_{1: i-1}\right)\right)$ must be found. Putting all together we yield $\mathcal{O}\left(B_{M}+2^{d} d^{2} B_{M}^{1 / d}\right)$ the generating one sample. As above we assume $h=h^{*}$, substitute $B_{M} \approx V_{M} / h^{d}$, and omit $V_{M}$ in order to derive $\mathcal{O}\left(\rho(d)^{-d /(d+4)} N^{d /(d+4)}+2^{d} d^{2} \rho(d)^{-1 /(d+4)} N^{1 /(d+4)}\right)$. As Step 1 needs to be carried out only once and as $\rho(d)^{-1 /(d+4)}$ is small compared to $2^{d} d^{2}$ we obtain approximately $\mathcal{O}\left(2^{d} d^{2} N^{(d+5) /(d+4)}\right)$ for generating $N$ samples. Finally, we remark that $N$ evaluations are negligible compared to generating $N$ samples. 


\section{REFERENCES}

Asmussen, S. (2003), Applied Probability and Queues, New York: Springer.

Geweke, J. (1989), "Bayesian Inference in Econometric Models using Monte Carlo Integration," in Econometrica, 57, 1317-1339.

Givens, G. H., and Raftery, A. E. (1996), "Local Adaptive Importance Sampling for Multivariate Densities With Strong Nonlinear Relationships," in Journal of American Statistical Association, 91, 132-141.

Glasserman, P., and Kou, S.-G. (1995), "Analysis of an Importance Sampling Estimator for Tandem Queues," in ACM Transactions on Modeling and Computer Simulation, 5, $22-42$.

Glasserman, P., Heidelberger, P., and Shahabuddin, P. (1999), "Asymptotically optimal importance sampling and stratification for pricing path-dependent options," in Mathematical Finance, 9, 117-152.

Glasserman, P. (2004), Monte Carlo Methods in Financial Engineering, New York: Springer.

Glynn, P. W., and Iglehart, D. L. (1989), "Importance Sampling for Stochastic Simulations," in Management Science, 35, 1367-1392.

Kim, Y. B., Roh, D. S., and Lee, M. Y. (2000), "Nonparametric Adaptive Importance Sampling For Rare Event Simulation," in Winter Simulation Conference Proceedings, Vol. 1, 767-772.

Kollman, C., Baggerly, K., Cox, D., and Picard, R. (1999), "Bayesian Inference in Econometric Models using Monte Carlo Integration," in Annals of Applied Probability, 9, 391-412.

Lazowska, E. D. (1984), Quantitative System Performance, Computer System Analysis Using Queuing Network Models, Prentice Hall.

Matsumoto, M., and Nishimura, T. (1998), "Mersenne Twister: A 623-Dimensionally Equidistributed Uniform Pseudo-Random Number Generator," in ACM Transactions on Modeling and Computer Simulations, 8, 3-30.

Musso, M., Oudjane, N., and Le Gland, F. (2001), "Improving Regularised Particle Filters," in Sequential Monte Carlo Methods in Practice, eds. A. Doucet, N. de Freitas and N. Gordon, New York: Springer.

Oh, M. S., and Berger, J. (1992), "Adaptive Importance Sampling in Monte Carlo Integration," in Journal of Statistical Computation and Simulation, 41, 143-168.

- (1993), "Integration of Multimodal Functions by Monte Carlo Importance Sampling," in Journal of American Statistical Association, 88, 450-456. 
Raftery, A. E., Givens, G. H., and Zeh, J. E. (1995), "Inference from a Deterministic Population Dynamics Model for Bowhead Whales," in Journal of American Statistical Association, 90, 402-430.

Robert, C. P., and Casella, G. (2004), Monte Carlo Statistical Methods, New York: Springer.

Rubinstein, R. Y. (1981), Simulation and the Monte Carlo Method, New York: Wiley.

Rubinstein, R. Y. (2007), "How to Deal with the Curse of Dimensionality of Likelihood Ratios in Monte Carlo Simulation," unpublished manuscript.

Scott, D. W. (1992), Multivariate Density Estimation, New York: Wiley.

Stadler, J. S., and Roy, S. (1993), "Adaptive Importance Sampling," in IEEE journal on selected areas in communications, 11, 309-316.

Terrell, G. R., and Scott, D. W. (1985), "Oversmoothed Nonparametric Density Estimates," in Journal of American Statistical Association, 80, 209-214.

West, M. (1992), "Modelling with Mixtures," in Bayesian Statistics 4, eds. J.M. Bernardo et al., Oxford UK: Oxford University Press, 503-524.

- (1993), "Approximating Posterior Distributions by Mixtures," in Journal of Royal Statistical Society, 55, 409-422.

Zhang, P. (1996), "Nonparametric Importance Sampling," in Journal of American Statistical Association, 91, 1245-1253.

Zlochin, M., and Baram, Y. (2002), "Efficient nonparametric importance sampling for Bayesian learning," in Neural Networks 2002, 2498-2502.

TABLES AND FIGURES 


\begin{tabular}{lc|cc|cc|cc}
\hline & & $N=1,000$ & & $N=5,000$ & & $N=10,000$ & \\
Method & $d$ & RE & Time & RE & Time & RE & Time \\
\hline \hline MC & 1 & 1.0 & 1 & 1.0 & 9 & 1.0 & 16 \\
IS & 1 & 1.5 & 3 & 1.8 & 16 & 1.6 & 31 \\
NIS & 1 & 1.6 & 13 & 1.8 & 28 & 1.7 & 50 \\
NIS+/- & 1 & 25.0 & 13 & 57.3 & 24 & 51.3 & 40 \\
\hline MC & 4 & 1.0 & 4 & 1.0 & 20 & 1.0 & 45 \\
IS & 4 & 5.0 & 7 & 5.2 & 38 & 4.2 & 80 \\
NIS & 4 & 3.1 & 112 & 4.0 & 234 & 3.8 & 408 \\
NIS+/- & 4 & 9.1 & 105 & 26.0 & 195 & 22.0 & 326 \\
\hline MC & 8 & 1.0 & 13 & 1.0 & 60 & 1.0 & 121 \\
IS & 8 & 18.6 & 20 & 23.0 & 104 & 26.3 & 209 \\
NIS & 8 & 7.8 & 600 & 17.4 & 1460 & 5.4 & 4020 \\
NIS+/- & 8 & 7.5 & 572 & 30.2 & 1290 & 37.4 & 2170 \\
\hline
\end{tabular}

Table 1: Simulation results for Example 1. All figures are computed/averaged over 100 independent runs.

\begin{tabular}{l|ccc}
\hline Method & $\begin{array}{c}N=1,000 \\
\text { Time }(\mathrm{ms})\end{array}$ & $\begin{array}{c}N=5,000 \\
\text { Time }(\mathrm{ms})\end{array}$ & $\begin{array}{c}N=10,000 \\
\text { Time }(\mathrm{ms})\end{array}$ \\
\hline \hline MC & 1.8 & 9.0 & 17.8 \\
CDIS & 6.0 & 27.8 & 54.5 \\
NIS & 13.7 & 29.2 & 48.9 \\
NSIS & 14.1 & 31.1 & 52.1 \\
\hline
\end{tabular}

Table 2: CPU times for the option pricing example (Example 2) averaged over 1,000 independent runs.

\begin{tabular}{lc|cc|cc}
\hline Method & $N$ & $\begin{array}{c}\varphi_{1} \\
a=.75\end{array}$ & $a=3.5$ & $\begin{array}{c}\varphi_{2} \\
\end{array}$ & \\
\hline \hline NSIS & 1,250 & 1.59 & 2.89 & 0.58 & $3=3.5$ \\
GAIS & 1,250 & 0.02 & 3.45 & 0.30 & 1.11 \\
LAIS & 1,250 & 0.75 & 0.99 & 1.92 & 0.58 \\
\hline NSIS & 5,000 & 8.08 & 4.50 & 9.21 & 5.09 \\
GAIS & 5,000 & 5.88 & 0.67 & 0.96 & 0.36 \\
LAIS & 5,000 & 3.45 & 1.30 & 2.63 & 0.42 \\
\hline NSIS & 10,000 & 9.38 & 4.75 & 11.06 & 5.77 \\
\hline
\end{tabular}

Table 3: Relative efficiency of NSIS, GAIS, and LAIS compared to SIS for Example 3. Figures for NSIS are computed over 1,000 independent runs. Figures for GAIS and LAIS are reprocessed from Table 2 in Givens and Raftery (1996).

\begin{tabular}{l|cc|cc|ccc|cc}
\hline & \multicolumn{2}{|c|}{$K=5$} & & \multicolumn{2}{|c|}{$K=10$} & & \multicolumn{2}{|c}{$K=30$} & \\
Method & $\mathrm{RE}$ & $\mathrm{CV}$ & $\mathrm{RE}$ & $\mathrm{CV}$ & $\mathrm{RE}$ & $\mathrm{CV}$ & $\mathrm{RE}$ & $\mathrm{CV}$ \\
\hline \hline MC & 1.0 & .001 & 1.0 & .14 & - & - & - & - \\
IS & .3 & .01 & 19.8 & .03 & - & .08 & - & .24 \\
NIS & .2 & .02 & 58.4 & .02 & - & .03 & - & .09 \\
\hline
\end{tabular}

Table 4: Results for the spam filter queueing application (single server case). Relative efficiency and coefficient of variation for the estimates of the probability that the queue length reaches level $K$. All figures are computed over 100 independent runs with 1 Mio. busy periods in each run. 


\begin{tabular}{|c|c|c|c|c|c|c|}
\hline Motbod & $K=4$ & CV & $K=6$ & $C \mathrm{~V}$ & $K=8$ & $C \mathrm{~V}$ \\
\hline$\overline{\overline{\mathrm{MC}}}$ & $\overline{1.0}$ & .007 & $\overline{10}$ & .044 & 1.0 & .34 \\
\hline IS & 3.7 & .003 & 7.0 & .017 & 53.5 & .046 \\
\hline NIS & 2.6 & .004 & 24.3 & .009 & 184.6 & .025 \\
\hline
\end{tabular}

Table 5: Results for the spam filter queueing application (dual server case). Relative efficiency and coefficient of variation for the estimates of the probability that the queue length reaches level $K$. All figures are computed over 100 independent runs with 1 Mio. busy periods in each run.
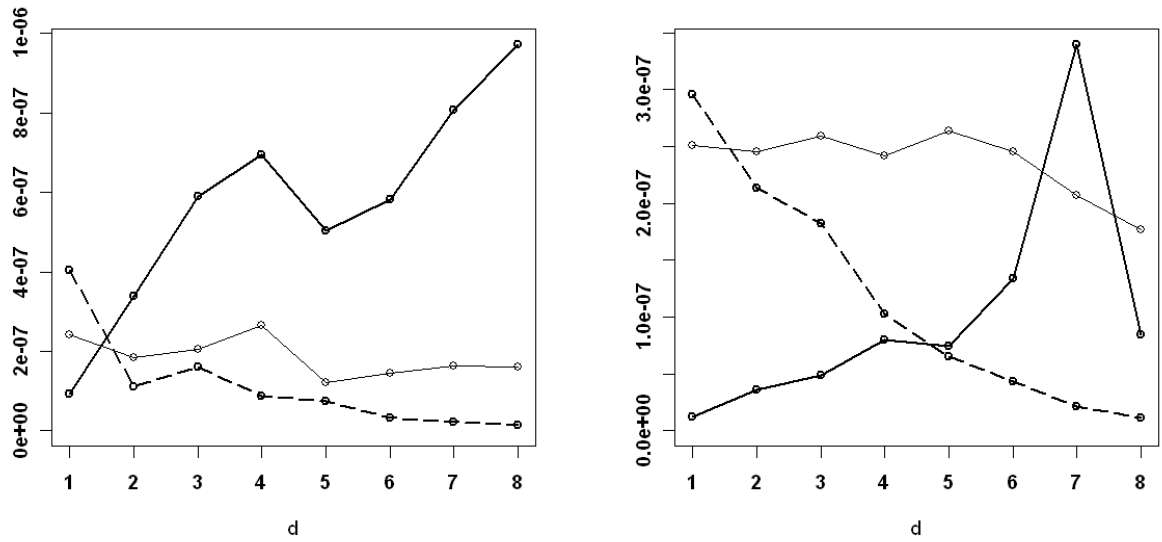

Figure 1: Computational efficiency (measured by MSE $\times$ computation time) of MC (solid line), IS (dashed line), and NIS $+/$ - (heavy line) for $N=1,000$ (left), and $N=10,000$ (right) for Example 1. All figures are computed/averaged over 100 independent runs.
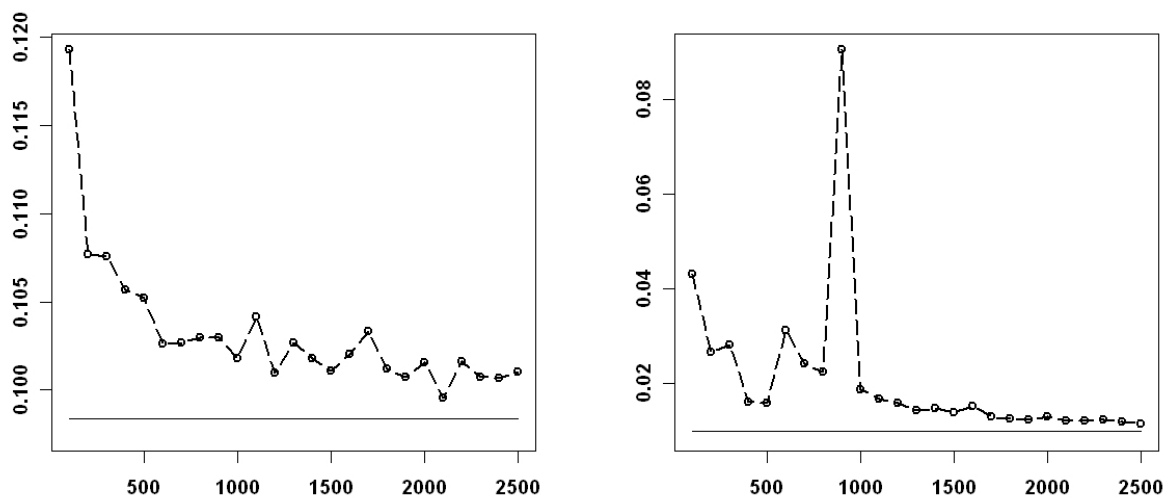

Figure 2: Convergence of NIS variance towards optimal IS variance for $d=1$ (left) and $d=4$ (right) for Example 1. All figures are computed over 10,000 independent runs. 

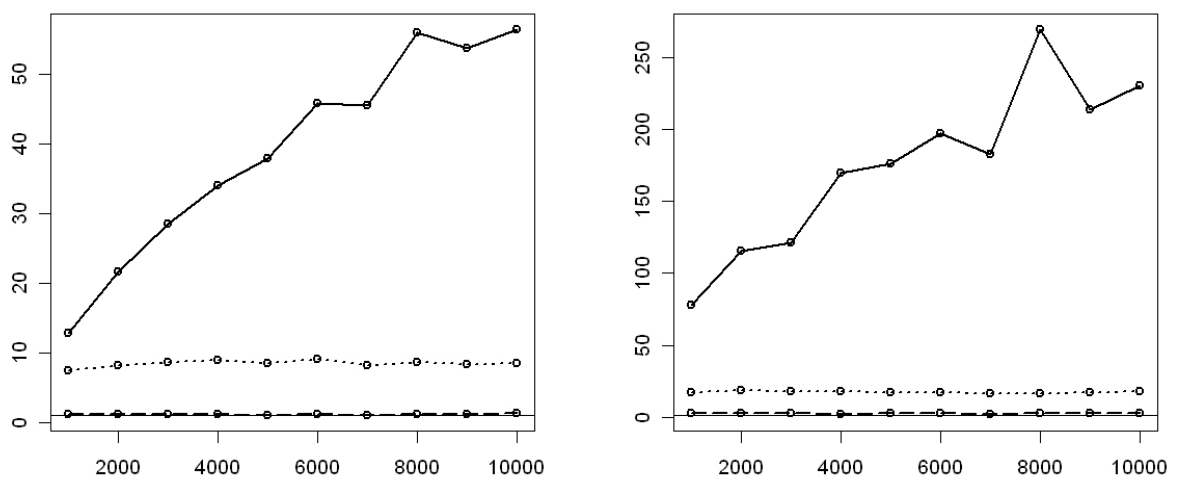

Figure 3: Relative efficiency of CDIS (dotted line), NIS (heavy line), NSIS (dashed line), and crude MC (solid line) for Example 2 (strike $K_{1}$ (left), strike $K_{2}$ (right)) and 1,000 $\leq N \leq 10,000$. All figures are computed over 1,000 independent runs.

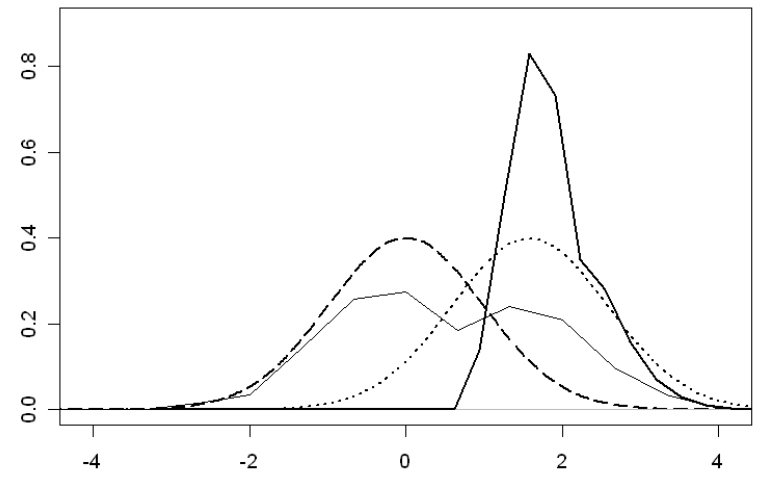

Figure 4: Standard normal distribution (dashed line), optimally shifted normal distribution (dotted line), linear blend frequency polygon estimates $(N=5,000)$ of the optimal proposals $q_{\varphi}^{\text {SIS }}$ (solid line), and $q_{\varphi}^{\text {IS }}$ (heavy line) for Example 2.
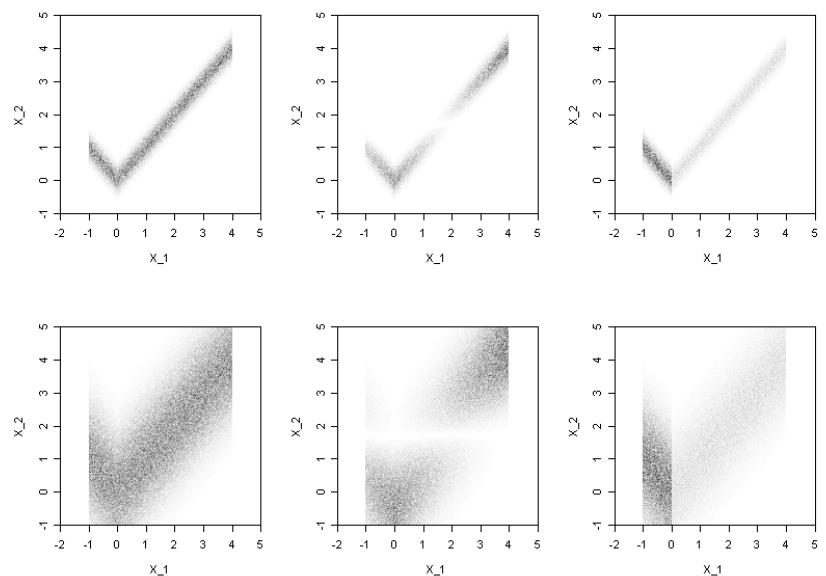

Figure 5: Example 3: The upper plots are for the case $a=.75$ and the lower plots for $a=3.5$. From left to right we have density $p\left(x_{1}, x_{2}\right)$ and the optimal proposals $q_{\varphi_{1}}^{\mathrm{SIS}}$ and $q_{\varphi_{2}}^{\mathrm{SIS}}$. 

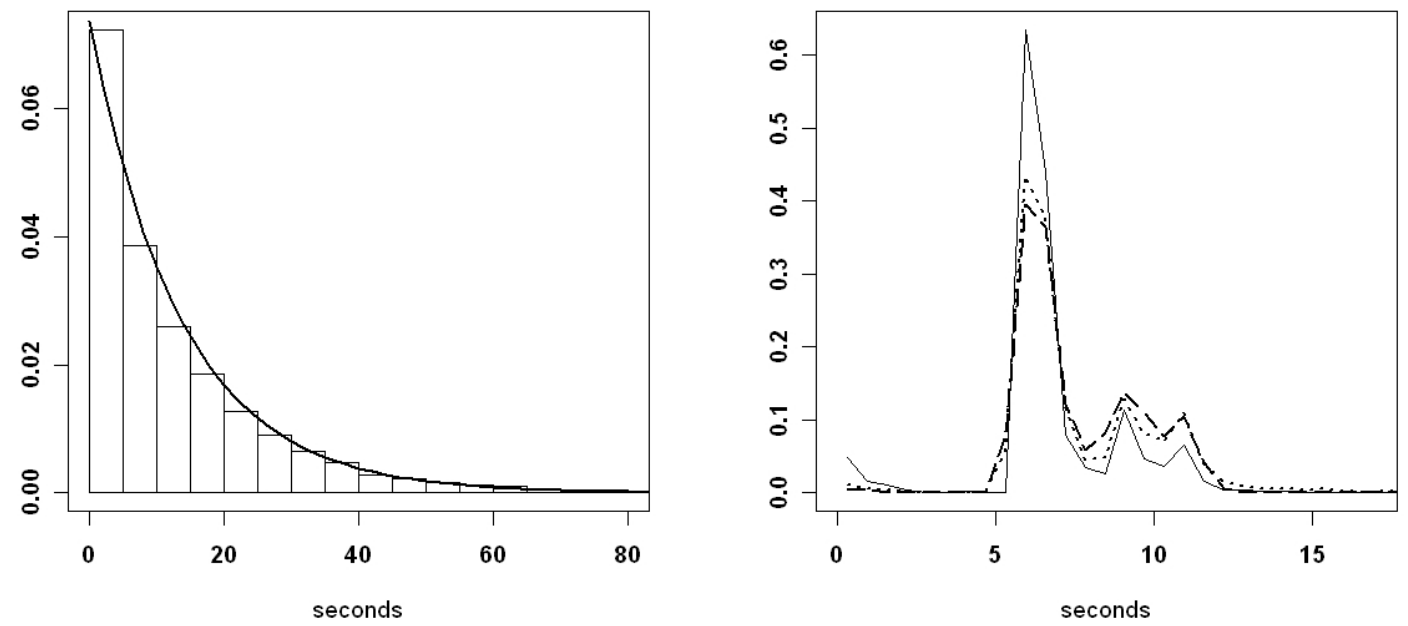

Figure 6: Spam filter application: Histogram of the empirical interarrival times and Exponential distribution with parameter .074 (left). Linear blend frequency polygon estimates of the service time distribution (solid line) and of the optimal proposal $q_{\varphi}^{\text {opt }}$ for the single server (dotted line) and dual server (dashed line) case for $K=10$ (right).

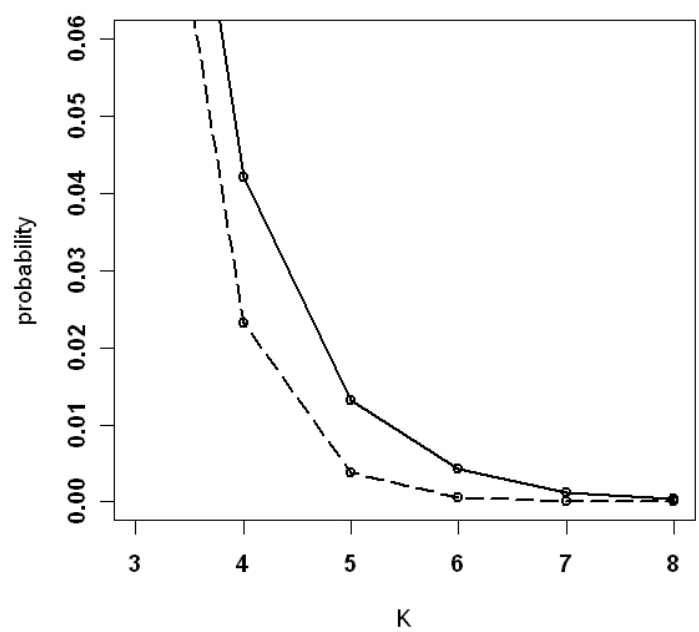

Figure 7: Results for spam filter application: Estimated probabilities of the queue length to reach level $K$ for single server (heavy line) and dual server (dashed line) case. 